\title{
Imaging tumor acidosis: a survey of the available techniques for mapping in vivo tumor $\mathrm{pH}$
}

\author{
Annasofia Anemone $^{1} \cdot$ Lorena Consolino $^{1} \cdot$ Francesca Arena $^{2,3} \cdot$ Martina Capozza $^{3}$ - Dario Livio Longo ${ }^{1,2}$
}

Published online: 14 February 2019

(C) The Author(s) 2019

\begin{abstract}
Cancer cells are characterized by a metabolic shift in cellular energy production, orchestrated by the transcription factor HIF- $1 \alpha$, from mitochondrial oxidative phosphorylation to increased glycolysis, regardless of oxygen availability (Warburg effect). The constitutive upregulation of glycolysis leads to an overproduction of acidic metabolic products, resulting in enhanced acidification of the extracellular $\mathrm{pH}(\mathrm{pHe} \sim 6.5)$, which is a salient feature of the tumor microenvironment. Despite the importance of $\mathrm{pH}$ and tumor acidosis, there is currently no established clinical tool available to image the spatial distribution of tumor $\mathrm{pHe}$. The purpose of this review is to describe various imaging modalities for measuring intracellular and extracellular tumor $\mathrm{pH}$. For each technique, we will discuss main advantages and limitations, $\mathrm{pH}$ accuracy and sensitivity of the applied $\mathrm{pH}$-responsive probes and potential translatability to the clinic. Particular attention is devoted to methods that can provide $\mathrm{pH}$ measurements at high spatial resolution useful to address the task of tumor heterogeneity and to studies that explored tumor $\mathrm{pH}$ imaging for assessing treatment response to anticancer therapies.
\end{abstract}

Keywords $\mathrm{pH}$ imaging · Tumor acidosis · Magnetic resonance imaging · Chemical Exchange Saturation Transfer (CEST) imaging $\cdot$ Iopamidol $\cdot \mathrm{pH}$-responsive probes

\section{Introduction}

Solid tumors are characterized by a highly heterogeneous microenvironment, resulting from the combination of poor vascular perfusion and regional hypoxia [1]. Metabolic adaptation represents a canonical response of tumor cells to survive, orchestrated by the transcription factor HIF- $1 \alpha$, which modulates genes involved in angiogenesis, glycolysis, proliferation, and metastasis. This metabolic shift is characterized by elevated glycolysis and lactate production, regardless of oxygen availability (Warburg effect) [2,3]. The constitutive upregulation of

Dario Livio Longo

dario.longo@unito.it; dariolivio.longo@cnr.it

1 Molecular Imaging Center, Department of Molecular Biotechnology and Health Sciences, University of Turin, Via Nizza 52, Turin, Italy

2 Institute of Biostructures and Bioimaging (IBB), Italian National Research Council (CNR), Via Nizza 52, Turin, Italy

3 Center for Preclinical Imaging, Department of Molecular Biotechnology and Health Sciences, University of Turin, Via Ribes 5, Colleretto Giacosa, Italy glycolysis leads to the exaggerated generation of metabolites, including acidic products such as lactate and protons that, upon accumulation in the cytoplasm, might result in intracellular acidosis. Therefore, tumor cells require additional activities in order to maintain an intracellular $\mathrm{pH}$ (pHi) compatible with the biochemical processes typical of cells characterized by high proliferation rates $[4,5]$. This task is taken up by several and redundant families of proton transporters that excrete lactate molecules and protons into the extracellular-extravascular compartment, which induces extracellular acidification, resulting in the reversed extracellular $\mathrm{pH}(\mathrm{pHe})$ gradient in tumors in comparison to healthy tissues [6].

Clinical investigations support the view that the acidic microenvironment in tumors results in less favorable prognosis associated with increased metastatic potential and resistance to chemo- and radiotherapy [7]. Therefore, acid production (or extracellular acidification) is even more important than the altered glucose metabolism [8,9]. Because survival in the tumor microenvironment depends on the control of $\mathrm{pH}$, interference with $\mathrm{pH}$ regulating systems is considered a relevant therapeutic goal [10]. Thus, extracellular acidosis of solid tumors acquires a prognostic value and therapeutic 
manipulation of tumor acidosis might represent a novel strategy for a successful treatment of cancer [11, 12].

On the basis of the above considerations, the need for advanced medical imaging approaches, based on mapping of tumor pHe that allows early detection of treatment effect upon the impairment of tumor $\mathrm{pH}$ dynamics, is urgently needed. The development/exploitation of imaging-based procedures able to quantitatively measure tumor $\mathrm{pHe}$ may provide noninvasive additional information to currently used nuclear medicine techniques $\left({ }^{18} \mathrm{~F}-\mathrm{FDG} / \mathrm{PET}\right)$ for cancer assessment. If $\mathrm{pH}$ regulation is an essential component of tumor cell survival and its impairment may halt proliferation of the primary tumor and formation of metastases, a tool able to noninvasively quantify tumor $\mathrm{pHe}$ appears to have optimal chances to be translated to the clinic.

This article surveys the imaging-related methods that have been proposed, and validated in vivo, for measuring tumor acidosis, including magnetic resonance imaging (MRI) and spectroscopy (MRS), nuclear medicine (positron emission tomography, PET), electron paramagnetic resonance (EPR), optical imaging (OI), and photoacoustic imaging (PAI). For each method, the basic principles are described together with the most representative $\mathrm{pH}$-sensitive probes and approaches that have been exploited for attaining in vivo tumor $\mathrm{pH}$ maps.

\section{Magnetic resonance imaging methods}

\subsection{Magnetic resonance spectroscopy}

Magnetic resonance spectroscopy (MRS) has been proposed in the early days of in vivo nuclear magnetic resonance (NMR) applications as a direct approach to the detection and quantification of metabolites in living tissues. In the oncological field, MRS allows the assessment of abnormal metabolic profiles that may act as useful prognostic biomarkers. In addition to metabolites, MRS has also been exploited for assessing $\mathrm{pHi}$ and $\mathrm{pHe}$ compartments of tumors cells by combining acceptable sensitivity threshold with spatial resolution. Intense efforts have been devoted to design suitable $\mathrm{pH}$ reporters with the aim of satisfying the criteria of favorable pharmacokinetics, $\mathrm{pKa}$ suitable for the physiological $\mathrm{pH}$ range, good sensitivity, and low toxicity. The measurement of $\mathrm{pH}$ by MRS is based on the chemical shift difference between a $\mathrm{pH}$ dependent resonance and a $\mathrm{pH}$-independent peak taken as reference. ${ }^{31} \mathrm{P},{ }^{1} \mathrm{H}$, and ${ }^{19} \mathrm{~F}$ have been the most investigated NMR active nuclei for selecting $\mathrm{pH}$-dependent resonances containing species.

\subsubsection{MRS $-{ }^{31} \mathrm{P}$}

${ }^{31} \mathrm{P}$ MRS has been the first technique applied for measuring $\mathrm{pH}$ in tumors. Phosphorus-31 is $100 \%$ abundant and several phosphorus-based metabolites are intracellularly present at a concentration of $0.1-5.0 \mathrm{mM}$. Among them, inorganic phosphate $(\mathrm{Pi})$ is routinely used for measuring $\mathrm{pH}$ in vivo due to the dependence of its chemical shift on $\mathrm{pH}$ changes in the physiological range ( $\mathrm{pKa} \sim 6.8$ ). As $\mathrm{pH}$-independent reference peaks, the signals from endogenous nucleoside triphosphates (NTP) and phosphocreatine (PCr) are usually considered. Concerns regarding the use of different reference spectra on $\mathrm{pH}$ determination have been raised; however, a recent comparative study involving healthy volunteers and patients with non-Hodgkin's lymphoma demonstrated that the direct $\mathrm{Pi}-\alpha$ ATP method is more reliable for measuring $\mathrm{pH}$ in tumors, showing low variation among patients and reasonable repeatability [13]. Considering that intracellular $\mathrm{Pi}$ is present at a concentration of $2-3 \mathrm{mM}$, whereas in the extracellular compartment is present at a concentration of $1 \mathrm{mM}$; it can be calculated that, for an extracellular volume below $55 \%$, most of the Pi-MRS-based signal is coming from the intracellular compartment, thus indicating that the observed shift of $\mathrm{Pi}$ is essentially a reporter of intracellular $\mathrm{pH}$ [14]. This statement has been validated in vivo by exploiting the intracellular $\mathrm{pH}$ reporter 2-deoxyglucose (2DG), that is phosphorylated to 2DG6P and accumulates within cells overcoming the glycolytic process [15]. ${ }^{31} \mathrm{P}-\mathrm{MRS}$ of fibrosarcoma xenograft tumors revealed a good correspondence between $\mathrm{pH}$ values obtained from Pi and from 2DG6P measurements, confirming that PiMRS measurements definitely report on intracellular $\mathrm{pH}$. To supply the lack of extracellular $\mathrm{pH}$ reporter probes for ${ }^{31} \mathrm{P}$ MRS, exogenous phosphonate agents have also been developed. Despite the fact that several extracellular phosphonatebased probes showed good characteristics [16], most in vivo applications have historically involved the use of 3aminopropylphosphonate (3-APP). This compound shows a $\mathrm{pKa}$ in the physiological range and accurately reports the $\mathrm{pHe}$ with little influence of temperature and ion effect [17]. Therefore, simultaneous acquisition of Pi and 3-APP can be combined for assessing the $\mathrm{pH}$ values within tissue compartments and quantitative parameters can be extrapolated for an extensive characterization [18]. ${ }^{31} \mathrm{P}-\mathrm{MRS}$ measurements elucidated in vivo the concept of the cellular $\mathrm{pH}$ gradient of tumors, indicating that intracellular $\mathrm{pH}$ in tumor is usually more alkaline in comparison to normal tissue, whereas extracellular $\mathrm{pH}$ is generally more acidic. This peculiar information has been exploited in several studies aiming at reverting the acidic-base $\mathrm{pH}$ gradient as a potential approach for treating cancer. This idea is based on the fact that the kinetic uptake of drugs strongly depends on their ionization state in relation to a specific $\mathrm{pHi} / \mathrm{pHe}$ condition [19]. Several in vivo investigations showed increased cytotoxic activity of chemotherapeutic drugs as mitoxantrone and doxorubicin upon induced tumor alkalinization with sodium bicarbonate, which raises the extracellular pH of 0.4-0.8 units. [20, 21] Moreover, inhibitors of mitochondrial metabolism in combination with 
hyperglycemic conditions induced selective acidification of human melanoma xenografts, with a significant decrease of both intra and extracellular $\mathrm{pH}$ [22]. Furthermore, ${ }^{31} \mathrm{P}-\mathrm{MRS}$ approach was recently used in a mouse model to evaluate early intracellular $\mathrm{pH}$ changes upon antiangiogenic treatment of recurrent glioblastoma [23]. This approach can therefore provide assessments of both intra- and extracellular tumor $\mathrm{pH}$ by combining endogenous and exogenous $31 \mathrm{P}-$ containing molecules. However, the potential neurotoxicity of 3-APP (analog of the $\gamma$-aminobutyric acid neurotransmitter) in the presence of compromised blood brain barriers is a concern for human use and the low spatial resolution and long acquisition times combined with the requirement of dedicated coils limit its application in vivo.

\subsubsection{MRS $-{ }^{19} \mathrm{~F}$}

${ }^{31} \mathrm{P}$-MRS has 15 times less sensitivity in comparison to ${ }^{1} \mathrm{H}$ spectroscopy; conversely, sensitivity of ${ }^{19} \mathrm{~F}$ is reasonably close to proton spectroscopy $(83 \%)$ and its isotopic abundance is $100 \%$. The main advantage of ${ }^{19} \mathrm{~F}-\mathrm{MRS}$ for in vivo application relies on the minimal NMR background interference from endogenous signal and the large chemical shift range $(\sim$ $300 \mathrm{ppm}$ ) that allowed the development of several fluorinated probes able to report microenvironment changes of $\mathrm{pO}_{2}$, hypoxia, enzyme activity, and $\mathrm{pH}$ [24]. Aromatic molecules, such as the vitamin B6 analogue fluoropyridoxol, were reported for assessing $\mathrm{pH}$ in vivo thanks to the larger chemical shift response $(\sim 9.5 \mathrm{ppm})$ to changes in $\mathrm{pH}$ in comparison to fluoroalanine-based probes ( $2 \mathrm{ppm}$ ) [25]. Early studies demonstrated the capability of 6-fluoropyridoxol (6-FPOL) to simultaneously measure the dynamic changes of $\mathrm{pHe} / \mathrm{pHi}$ in perfused rat hearth with a time resolution of 2 min [24]. As the $\mathrm{pKa}(\sim 8.2)$ of 6-FPOL is not ideal, a novel membraneimpermeant CF3-modified 6-trifluoromethylpyridoxine with a $\mathrm{pKa}$ in the physiological range was designed. This new molecule was successfully detected in mammary and prostate tumor xenografts allowing the measurement of the extracellular $\mathrm{pH}$ with a sensitivity of $0.40 \mathrm{ppm} / \mathrm{pH}$ unit [26]. However, fluorophenols have the drawback of ion-binding, thus limiting their application in vivo. An additional ${ }^{19} \mathrm{~F}$-MRS probe, the fluoroaniline sulfonamide ZK-150471, has been validated as a valid $\mathrm{pH}$ reporter in different tumor xenograft models. The aromatic fluorine signal of ZK-150471 showed a chemical shift highly dependent on $\mathrm{pH}$, whereas the trifluoromethyl group served as the intramolecular $\mathrm{pH}$-independent reference. In vivo, ZK-150471 demonstrated to distribute only within the extracellular space of the perfused regions, showing good correspondence with microelectrodes $\mathrm{pHe}$ measurements [27]. Further, the capability of ZK-150471 to report pHe was compared with the ${ }^{31} \mathrm{P}$-MRS probe 3-APP in tumors. Although $\mathrm{pHe}$ values were not significantly different, the fluorinated probe has the advantage of a lower toxicity, thus allowing the administration of higher doses for investigating $\mathrm{pH}$ heterogeneity in tumors. The main limitations for in vivo applications are the relative instability of fluorinated probes and their nonspecific accumulation in tissues due to their hydrophobicity. To overcome these issues, new formulations based on the encapsulation of ${ }^{19} \mathrm{~F}$ compounds have been proposed. Promising results were obtained with PEGylated nanogels that showed variation in size in accordance with $\mathrm{pH}$ changes, in the range of 6.8-7.3, indicating that extracellular $\mathrm{pH}$ can be indirectly estimated from measuring the diameter of nanoprobes by ${ }^{19}$ F-MRS [28]. Similarly, an on/off strategy based on micelles encapsulating ${ }^{19} \mathrm{~F}$ containing species allowed the encoding of narrow $\mathrm{pH}$ transition $(0.25 \mathrm{pH}$ unit $)$ through barcode map generated from specific ${ }^{19} \mathrm{~F}$ signatures [29]. Despite the absence of a background signal that improves the specificity of this approach, the moderate sensitivity still limits the spatial resolution achievable by ${ }^{19} \mathrm{~F}-\mathrm{MRS}$, even at higher magnetic fields.

\subsubsection{MRS- ${ }^{1} \mathrm{H}$}

To overcome both ${ }^{31} \mathrm{P}$ - and ${ }^{19} \mathrm{~F}$-MRS issues, proton spectroscopy represents a valid alternative in terms of increased sensitivity due to its highest gyromagnetic ratio and a natural abundance of $99.98 \%$. These properties represent a great advantage for gaining in spatial resolution with reduced acquisition time but a technical challenge since the broad and intense peak arising from the bulk water molecules need to be nullified for observing the smaller resonances of the exploited probes. For instance, a ${ }^{1} \mathrm{H}-\mathrm{NMR} \mathrm{pH}$-sensitive probe was reported by Aime and coworkers, exploiting a paramagnetic complex of Ytterbium, a lanthanide ion able to induce large paramagnetic shifts of nearby nuclei [30]. This compound was shown in vitro to represent an excellent $\mathrm{NMR} p H$ indicator since the chemical shift separation between a selected pair of resonances is strongly $\mathrm{pH}$ dependent and not affected by changes in concentration or in ionic strength. Moreover, the NMR resonances of the paramagnetic complex cover a much wider chemical shift region than those of diamagnetic systems, providing higher $\mathrm{pH}$ sensitivity since the NMR resonances are easily distinguishable from that of endogenous molecules. Further details of the in vivo exploitation of the large ${ }^{1} \mathrm{H}$-chemical shift of paramagnetic metal complexes as $\mathrm{pH}$ indicators can be found in the PARACEST paragraph.

For measuring in vivo $\mathrm{pH}$ by ${ }^{1} \mathrm{H}-\mathrm{MRS}$, histidine and imidazole-containing molecules were initially considered due to (i) their $\mathrm{pKa}(\sim 7.0)$ close to the physiological values and (ii) their proton NMR peaks that resonate enough away from the very intense water signal. First studies exploited histidine, a precursor of imidazole, since the presence of two resonances that show a $\mathrm{pH}$-dependent chemical shift due to the protonation and deprotonation of the amine group [31]. Exploiting an oral histidine loading to increase the natural 
concentration of this molecule in the brain, they were able to report $\mathrm{pH}$ values in healthy human brains. The most significant results were achieved with 2-imidazole-1-yl-3ethoxycarbonyl propionic acid (IEPA) that demonstrated to remain well confined in the extracellular environment. H2IEPA shows a chemical shift that is highly dependent on $\mathrm{pH}$ with a titratable range of 7.8-8.9 ppm and a good $\mathrm{pH}$ accuracy of $0.1 \mathrm{pH}$ units, thus providing the first in vivo tumor $\mathrm{pHe}$ map [32]. This approach was further explored in a C6 glioblastoma multiforme model implanted in rats with a spatial resolution of $1 \times 1 \times 4 \mathrm{~mm}^{3}$ [33]. In this study, IEPA demonstrated that a more acidic $\mathrm{pH}$ occurs in tumors in comparison to normal tissues. In addition, this approach allowed mapping the distribution of IEPA, metabolic compounds, and lactate within the same acquisition experiment. Although it was expected a spatial correlation with lactate and proton concentration, a local mismatch between the two measurements was reported. This result was further elaborated in a subsequent paper devoted to the use of a new imidazole-derivative, the ( \pm )2-(imidazole-1yl)succinic acid (ISUCA, Fig. 1a) that demonstrated superior pharmacokinetic properties in comparison to IEPA and a chemical shift strongly dependent on $\mathrm{pH}$ changes, Fig. 1b, c [34]. Similarly, a voxel by voxel comparison showed a different spatial distribution of acidity and lactate in gliomas, suggesting that intra- and extracellular flow of protons might influence acidosis at distant sites from lactate accumulation. More recently, ISUCA was exploited to monitor the activity of the isoenzyme carbonic anhydrase IX (see Pastorikova this volume) that produces $\mathrm{H}^{+}$and $\mathrm{HCO}_{3}{ }^{-}$in the extracellular environment from the hydration of $\mathrm{CO}_{2}$, and is associated with the most aggressive forms of cancer [35]. This mechanism of acidification has been validated in vivo on colorectal tumor xenografts with different expression levels of CAIX. Interestingly, mapping of pHe with ISUCA revealed that high CAIX expression decreased the steady state $\mathrm{pH}$ of the extracellular compartment in comparison to low CAIX expressing tumors, whereas no evident differences in pHi measurements by ${ }^{1} \mathrm{H}-\mathrm{MRS}$ were observed (Fig. 1d, e).

Although the accuracy and sensitivity on reporting $\mathrm{pH}$ changes offered by this method, the poor spatial resolution and the long acquisition times markedly affect $\mathrm{pHe}$ estimation and represent a limitation for accurate quantification of $\mathrm{pHe}$ heterogeneity. Another concern on using imidazole-based compound was raised due to the alkalization effect of nonvolatile buffer that might affect $\mathrm{pH}$ measurements. Indeed, IEPA demonstrated buffering capabilities and chronic administration of IEPA influenced the extracellular acidity of tumors and reduced the formation of lung metastasis in an experimental model of prostate cancer [36]. In addition, the rapid elimination by renal clearance and the presence of only one $\mathrm{H} 2$ proton in IEPA and ISUCA limit the sensitivity of this approach for in vivo application. To overcome this issue, the design of diimidazole probes with higher polarity and double
$\mathrm{H} 2$ resonance intensity is currently under investigation, with promising results for in vivo pHe measurements [37].

\subsection{MRI relaxometry-based $\mathrm{pH}$-sensitive probes}

Magnetic resonance imaging (MRI) has been widely used as a routine diagnostic tool in modern clinical medicine. The majority of clinically used MRI contrast agents are paramagnetic gadolinium $\left(\mathrm{Gd}^{3+}\right)$ chelates, able to increase the signal intensity by shortening the longitudinal $\left(T_{1}\right)$ or the transversal $\left(T_{2}\right)$ relaxation times of water protons close to the region where the metal complex distributes [38-41].

pH-dependent paramagnetic metal complexes allow the evaluation of $\mathrm{pH}$ changes in extracellular tumor microenvironment by altering their $T_{1}$ MR relaxation $[42,43]$. The first approach to measure extracellular $\mathrm{pH}$ was described by Sherry and coworkers who exploited the protonation of the pendant arm of the $\mathrm{Gd}^{3+}$ complex of a tetraamide-based ligand. The changes in $T_{1}$ relaxation times reflect changes in the number of inner sphere water molecules which is determined by the protonated/deprotonated state of the ligand [44]. The in vivo application of this $\mathrm{pH}$-responsive agent was investigated with the tetraphosphonate gadolinium complex Gd-DOTA-4Amp in both C6 glioma and renal carcinoma [45, 46]. However, with this approach, changes in water proton relaxation times are dependent on both the concentration of the agent and its $\mathrm{pH}$ responsiveness; therefore, a direct readout of the solution $\mathrm{pH}$ is not possible. To overcome this limitation, a double injection of two similar contrast agents was proposed, one whose relaxivity is $\mathrm{pH}$ independent (Gd-DOTP) to estimate the concentration within the tissue of interest and the second one, with its relaxivity dependent on $\mathrm{pH}$. By setting up a ratiometric method (i.e., by dividing the measured estimates), it is possible to calculate the $\mathrm{pH}$. Unfortunately, the main drawbacks of this approach are as follows: (i) the administration of two contrast agents, (ii) one has to assume analogous pharmacokinetics and biodistribution of the two agents within the same anatomical region. A similar approach has been exploited following the simultaneous injection of two paramagnetic agents, in which the $\mathrm{pH}$-sensitive Gd-DOTA4Amp was mixed with the pH-insensitive $T_{2}{ }^{*}$ dysprosiumDOTP agent (Fig. 2) [47]. Although this protocol allowed the investigators to obtain high-resolution pHe maps using relatively standard doses $(0.2 \mathrm{mmol} \mathrm{Gd} / \mathrm{kg})$, still changes in $T_{2}{ }^{*}$ need to be measured before and after the injection of the agents, assuming no competing effects in $T_{1} / T_{2}{ }^{*}$ times between the two contrast agents. Moreover, a similar biodistribution of the two probes within the tumor region must be proven for correctly quantifying tumor $\mathrm{pHe}$. Other Gdbased agents have been investigated for mapping $\mathrm{pH}$ in a concentration-independent manner, but unfortunately without in vivo validation studies. A Gd-based complex covalently bound to an oligopeptide that undergoes $\mathrm{pH}$-dependent 


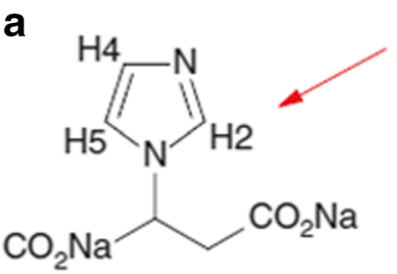

b

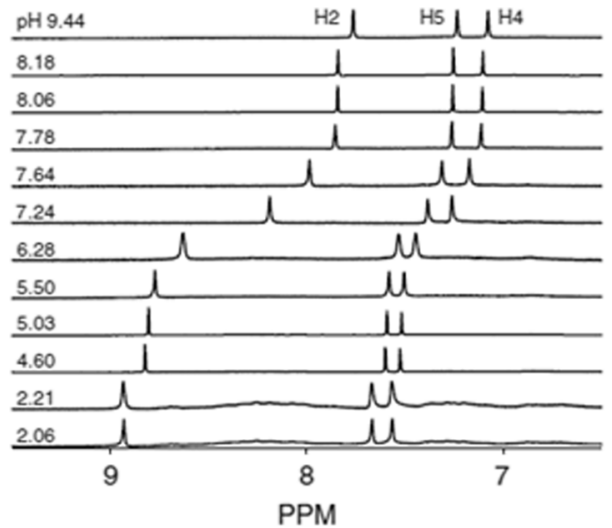

d
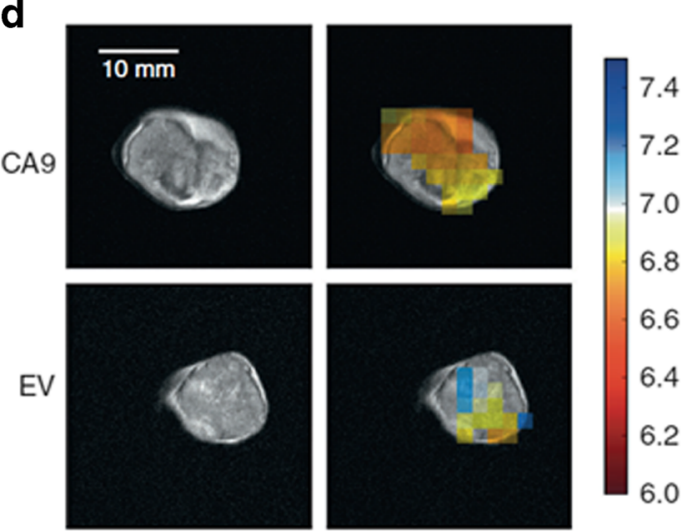

Fig. 1 MRS imaging of tumor $\mathrm{pH}$. Chemical formula of ISUCA and indications of proton resonances ( $\mathrm{pH}$-dependent $\mathrm{H} 2$, red arrow) on the imidazole ring (a). Plasmatic MR spectra of ISUCA at different $\mathrm{pH}$ values indicate the chemical shift dependency of $\mathrm{H} 2$ from $\mathrm{pH}$ changes (b). $\mathrm{pH}$ calibration curve of the chemical shift of $\mathrm{H} 2$ with changes in $\mathrm{pH}$ (c).

configuration changes resulting in $T_{1} / T_{2}$ relaxation variations. Also, Aime and coworkers have demonstrated in vitro that Gd-DO3Asa-loaded liposomes provide a method for measuring $\mathrm{pH}$ by ratioing the measured relaxivities at different magnetic field strengths, opening new ways for relaxometric approaches $[48,49]$. Other approaches, combining dual ${ }^{1} \mathrm{H}$ and

${ }^{19} \mathrm{~F}$ containing probes using the heteronuclear ${ }^{19} \mathrm{~F}$ signal to normalize the contrast enhancement values due to a $\mathrm{pH}$ sensitive Gd-complex, have also been investigated [50].

\subsection{MRI chemical exchange saturation transfer imaging}

Chemical exchange saturation transfer (CEST) imaging is a MRI contrast mechanism that allows noninvasive detection of
C

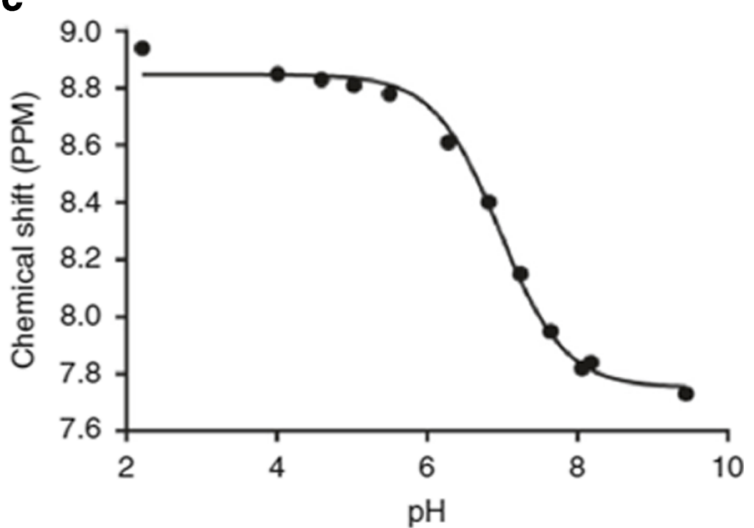

e

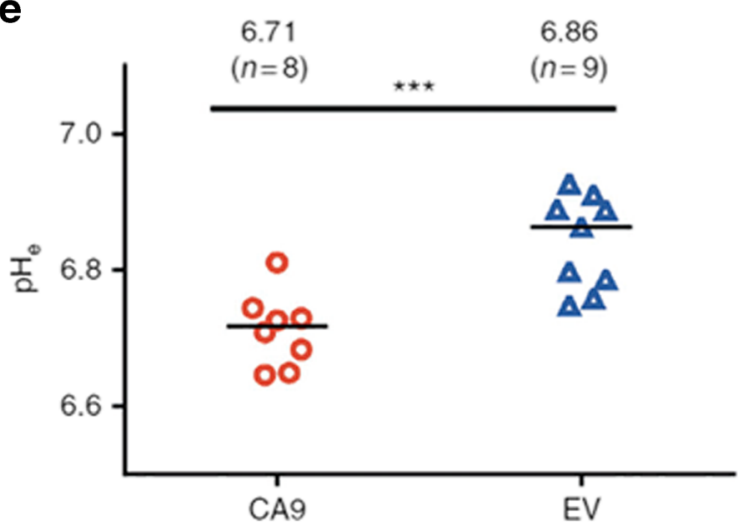

Parametric pHe map over-imposed on $T_{2}$-weighted MR images of high expressing CAIX (CA9) and low expression CAIX (EV) tumors (d) and median pHe value for CA9 and EV tumors (e). Adapted with permission from British Journal of Cancer 2018, 119, 622-630

molecules or microenvironmental tumor properties such as $\mathrm{pH}$, redox status, and enzymatic activity [51]. CEST method is based on the detection of changes of the water protons signal that decreases upon saturation magnetization at specific frequencies from an exchanging pool of protons, which can be from endogenous or exogenous sources. When RF pulses are applied, exchangeable protons from the contrast agent molecule become saturated and transfer their saturation to bulk water protons according to the chemical exchange rate, inducing a partial loss of net water signal. By applying a series of pulses, the saturation is transferred continuously to the water protons and is possible to detect the presence of molecules with mobile protons within MR images [52-54]. By recording the changes in the water signal intensity as a function of the applied RF pulses at different resonances (frequencies), one 

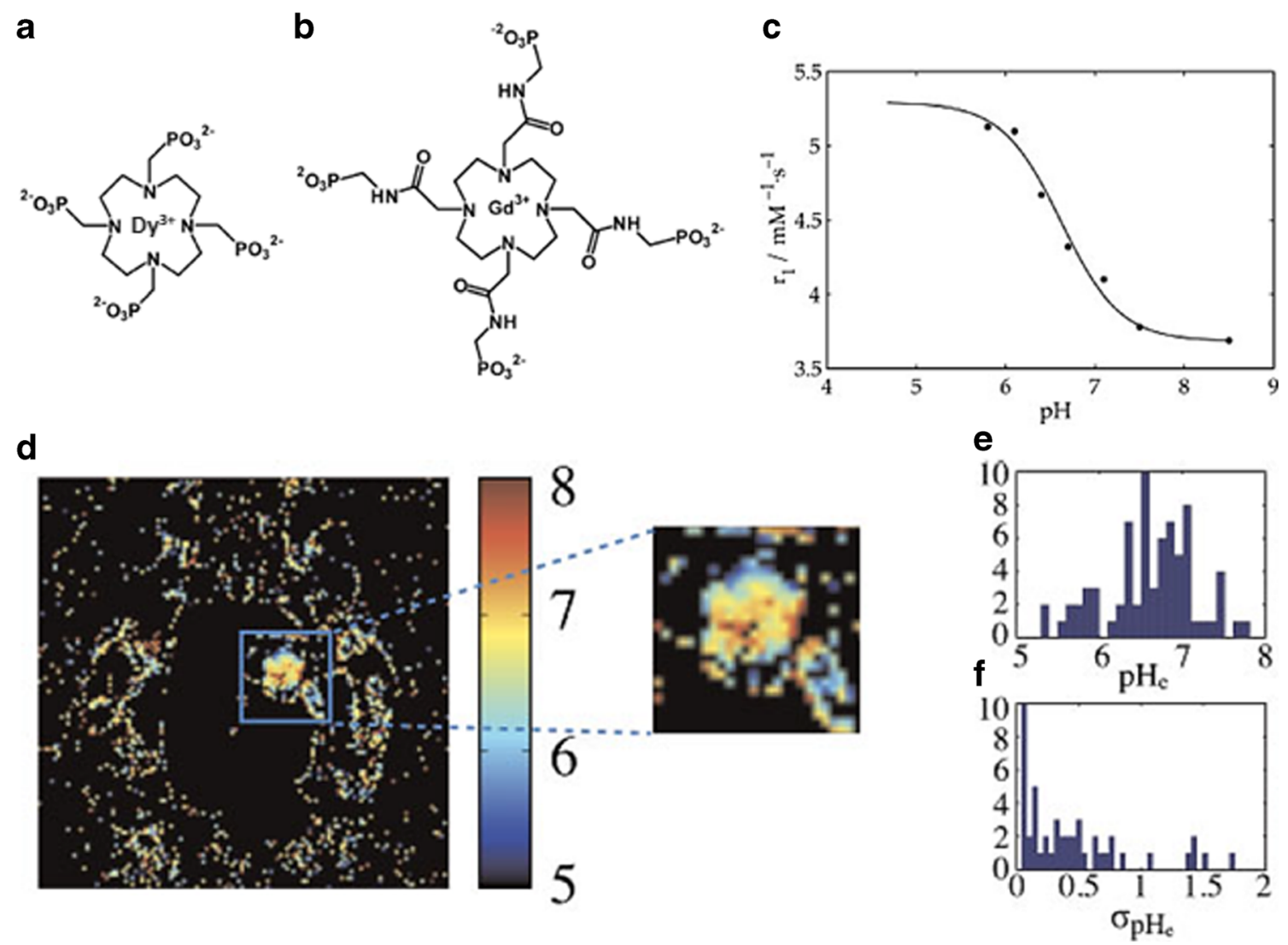

Fig. 2 MRI-based relaxometry imaging of tumor $\mathrm{pH}$. Chemical structure of the $\mathrm{pH}$-insensitive $T_{2}$ agent, Dy-DOTP (a) and of the $\mathrm{pH}$-sensitive $T_{1}$ agent, Gd-DOTA-4AmP (b). pH dependence of Gd-DOTA-4AmP relaxivity (c). Tumor pHe maps obtained following the injection of the

can obtain the so-called Z-spectrum that provides information on the exchanging molecule.

Proton exchange process depends on several factors, including concentration, temperature and, very often, $\mathrm{pH}$. Many agents show an exchange rate that is usually slower at low $\mathrm{pH}$ than at high $\mathrm{pH}$ due to the occurrence of basecatalyzed proton exchange [55]. To date, several $\mathrm{pH}-$ responsive CEST MRI agents have been developed and in general, CEST has some advantages compared to other imaging modalities. First, CEST MRI has a great spatial resolution (less than $1 \mathrm{~mm}$ ) that can provide tomographic images exploiting standard ${ }^{1} \mathrm{H}$ coils available in clinical MRI scanners. In addition, in contrast to MRI-relaxivity methods, CEST can take advantage of the ratiometric approach for ruling out the concentration provided that in the same agent there are two different pools of exchanging protons. On the other hand, it suffers of limited sensitivity as it needs millimolar concentrations of mobile protons. The MRI-CEST approach can be further subdivided according to the chemistry of the molecule containing the exchanging protons in DIACEST (diamagnetic molecules, including both endogenous and exogenous molecules) or PARACEST (paramagnetic molecules, mainly represented by metal complexes) agents cocktail of the two agents (d) and histograms of $\mathrm{pH}$ values (e) and standard deviations (f) within a region of interest. Reproduced with permission from NMR in Biomedicine 2011, 24, 1380-1391

$[56,57]$. The versatility of the CEST approach and probes enables different ways for measuring in vivo tumor acidosis.

\subsubsection{Endogenous DIACEST pH methods}

The chemical shift of typical endogenous diamagnetic exchangeable protons is within a few parts per million from bulk water protons $(0-4 \mathrm{ppm})$ and since the exchange is base-catalyzed, the exchange rate is lower at acidic $\mathrm{pH}$ values. Amide protons contained in endogenous proteins and peptides have been the first source of mobile protons to be imaged and investigated for obtaining $\mathrm{pH}$ measurements according to their CEST contrast dependence from $\mathrm{pH}$. This approach, called amide proton transfer (APT) imaging, has been exploited to obtain $\mathrm{pH}$ maps occurring in experimental models of brain stroke [58], since the reduced $\mathrm{pH}$ level in the ischemic region leads to a reduction in APT exchange rate, i.e., to a decrease of the CEST effect $[59,60]$. This endogenous $\mathrm{pH}$ mapping method has also been shown to detect changes in $\mathrm{pH}$ following ischemia damage, but not yet applied to map tumor $\mathrm{pH}$ [61]. APT $\mathrm{pH}$ mapping has some limitations, since it is not possible to distinguish between the intracellular and extracellular contribution of amide mobile protons; hence, the derived $\mathrm{pH}$ 
values are a weighted estimate of both intracellular and extracellular $\mathrm{pH}$. Secondly, the concentration of amide protons may vary in tumor; therefore, accurate $\mathrm{pH}$ values cannot be calculated. To overcome these limitations, Sun and colleagues have proposed and investigated different correction approaches to isolate the exchange rate (i.e., $\mathrm{pH}$-weighted) contribution in the human brain [62-65]. On the other hand, Bartha's group set up an amine/amide concentration-independent detection method (AACID) based on a ratiometric approach [66]. CEST effects originating at $2.75 \mathrm{ppm}$ for amine groups and at $3.5 \mathrm{ppm}$ for amide protons belonging to endogenous mobile peptides and proteins are ratioed (i.e., divided), allowing removal of the concentration term from the calculated $\mathrm{pH}$ values. With the AACID approach, they were able to detect in vivo intracellular $\mathrm{pH}$ changes after administration of different anticancer treatments in a glioblastoma murine model. Following the treatment with topiramate, an inhibitor of carbonic anhydrases, they observed a reduction of the intracellular tumor $\mathrm{pH}$ (corresponding to an increase in AACID values of 0.15 units) (Fig. 3) [67]. The method is sensitive enough to detect the slight changes in intracellular acidification of a implanted brain tumor following a single-dose administration of different drugs, such as lonidamine and dichloroacetate, targeting hexokinases and pyruvate dehydrogenase kinase, respectively $[68,69]$. More recently, the same group applied this approach to monitor the therapeutic effect of quercetin, a monocarboxylate transporter inhibitor, on intracellular tumor acidification [70]. Since this approach does not require the administration of exogenous agents, it can be readily translated to the clinic. However, the abovementioned limitations of mixing intracellular and extracellular contribution on the calculated $\mathrm{pH}$ still hold true.

\subsubsection{Exogenous DIACEST pH-responsive probes}

Exogenous DIACEST agents have mobile protons with a chemical shift in the range $0-10 \mathrm{ppm}$ from the water resonance. The main advantage in using exogenous agents relies on their capability to provide a net quantification of the extracellular tumor $\mathrm{pH}$, since following the extravasation from the leaky tumor vasculature they remain in the extracellular extravascular space and do not enter the intracellular compartment. Iodinated or X-ray contrast agents, following the clever intuition of Silvio Aime [71], gained a lot of attention as DIACEST pH-sensitive agents for several reasons, namely, (i) the chemical structure of these molecules contains exchangeable amide protons that can be selectively saturated and provide CEST contrast in the 4-6 ppm range, far enough from water for a selective irradiation even at the magnetic field of $3 \mathrm{~T}$; (ii) the CEST contrast is $\mathrm{pH}$ dependent and by applying a ratiometric approach, the $\mathrm{pH}$ measurement can be made concentration-independent; (iii) they are FDA-approved contrast agents used in the last 40 years for computed tomography (CT) investigations and have demonstrated a very high safety a

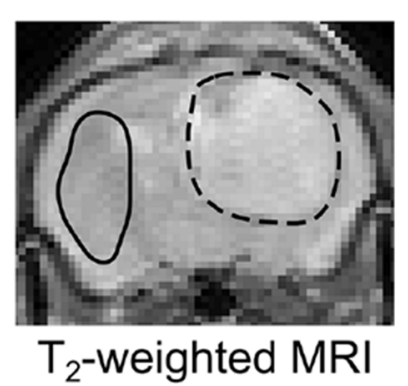

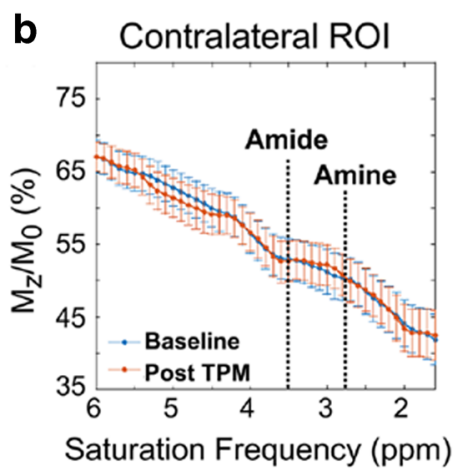
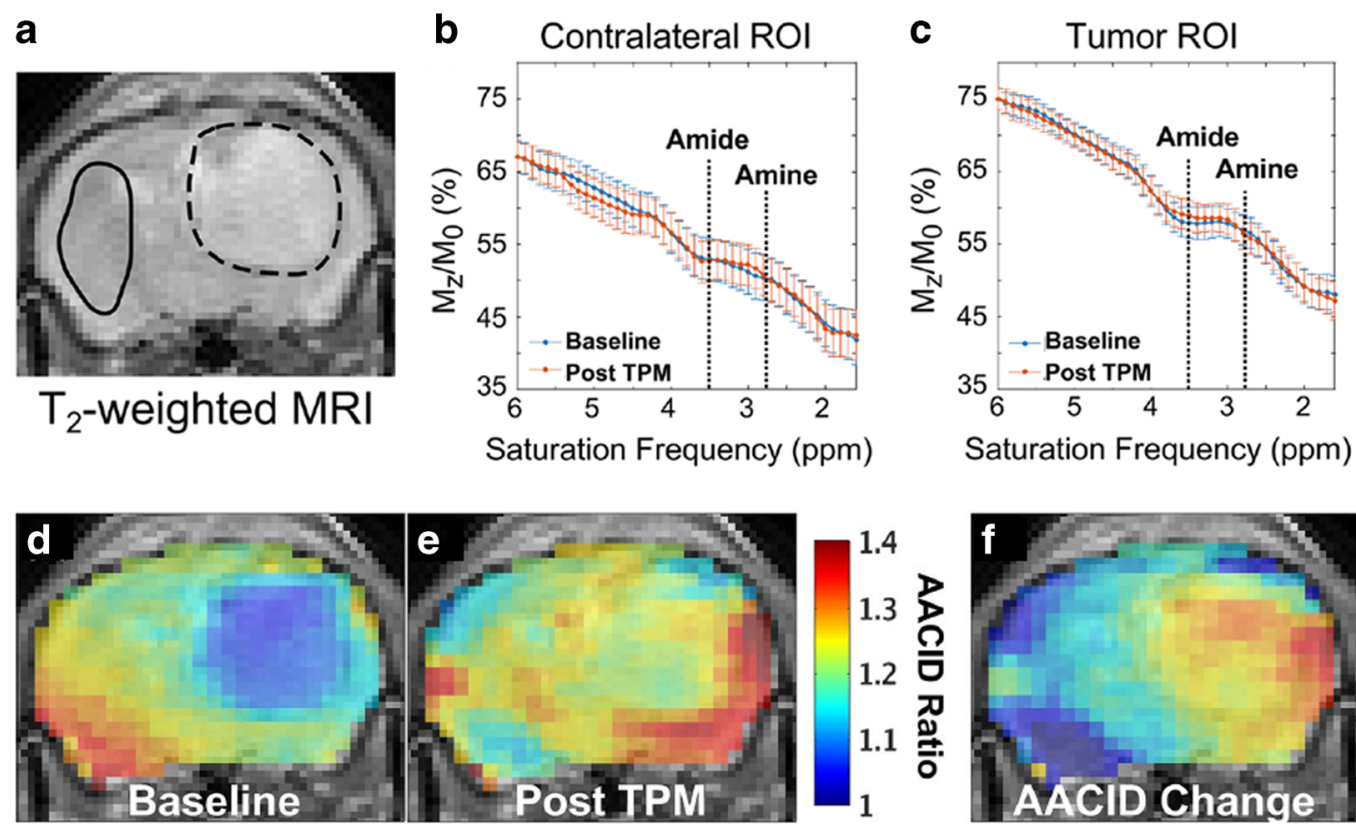

Fig. 3 MRI-CEST imaging of tumor $\mathrm{pH}$ using endogenous DIACEST approach. $T_{2}$-weighted MR image of a mouse brain with a U87 GBM tumor showing (a) region of interests (ROI) for the tumor region (dashed line) and for the contralateral region (solid line). CEST spectra from the contralateral (b) and from the tumor tissue (c) ROIs at baseline (blue) and $\sim 75 \mathrm{~min}$ (red) after administration of $120 \mathrm{mg} / \mathrm{kg}$ of topiramate (TPM).
The AACID measurement from the amine $(2.75 \mathrm{ppm})$ and the amide (3.5 ppm) signal allows the calculation of the AACID maps before and $\sim 75$ min after i.p. injection of TPM (d, e, respectively) and the calculation of the AACID change map showing tumor selective acidification (f). Adapted with permission from Journal of Neuro-Oncology 2016, 130, $465-472$ 
profile. Ongoing studies are also evaluating these molecules as alternatives to Gd-based agents for studying tumor perfusion [72, 73].

Iopamidol (Isovue $\AA$, Bracco Diagnostics) was the first CT agent investigated as a MRI-CEST contrast agent, due to the high number of mobile protons (three amide protons and five hydroxyl protons) [74]. In particular, Iopamidol has two amide groups that resonate at 4.2 and $5.5 \mathrm{ppm}$, endowed with a $\mathrm{pH}$ dependent exchange rate that allows to set up the proper ratiometric approach for measuring $\mathrm{pH}$. In fact, it has been shown to provide good $\mathrm{pH}$ sensitivity and high $\mathrm{pH}$ accuracy in the 5.5-7.9 range (Fig. 4) [75, 76]. While the first in vivo validation dealt with measuring renal $\mathrm{pH}$ and the assessment of $\mathrm{pH}$ changes in several models of kidney injuries [77, 78], subsequent investigations demonstrated the usefulness of this approach in providing accurate tumor $\mathrm{pHe}$ maps. Notably, the first in vivo validation of the relationship between tumor dysregulated glycolysis and acidosis was reported by combining MRICEST tumor pHe mapping with Iopamidol and PET imaging following ${ }^{18}$ F-FDG injection [79]. This work showed the feasibility of $\mathrm{pH}$ mapping using a clinical magnetic field strength of $3 \mathrm{~T}$ and using homogenates of murine tumors for building the $\mathrm{pH}$ calibration curve (Fig. 5a). In addition, we showed that the method can monitor tumor $\mathrm{pHe}$ changes following sodium bicarbonate treatment (Fig. 5b), which neutralizes tumor acidity. Our group provided a clear evidence of the relationship between tumor metabolism and acidosis since tumor regions with higher FDG uptake displayed lower pHe values in a HER2 positive breast cancer model (Fig. 5c). Thanks to the high spatial resolution attainable by the MRI modality, the obtained tumor pHe maps showed the occurrence of a large heterogeneity in tumor acidosis that was not possible to investigate so far with other imaging modalities (Fig. $5 \mathrm{~d}$, e), therefore providing complementary information to the gold standard ${ }^{18}$ F-FDG PET for assessing tumor metabolism. Furthermore, it has been found that in vivo MRI-CEST $\mathrm{pH}$ mapping is a valuable tool for monitoring the therapeutic effect of novel anticancer therapies targeting tumor metabolism [10, 11, 80]. Dichloroacetate (DCA) is a small mitochondria-targeting molecule that is able to revert the glycolytic phenotype by blocking the pyruvate conversion to lactate, thus potentially reducing the extracellular tumor acidification [81]. Breast tumor-bearing mice treated with DCA showed a marked increase of tumor $\mathrm{pHe}$ values just 3 days after DCA administration, indicating the capability of CEST $\mathrm{pH}$ mapping to monitor early treatment effects that were associated with the decrease of lactate production (Fig. 6) [82]. Moreover, this approach was able to detect the onset of the resistance to the DCA treatment after 15 days, when tumor $\mathrm{pHe}$ returned to more acidic values. Overall, these results highlight the relationship between glycolysis, lactate, and acidosis and how the overall picture can be well investigated using MRI-CEST $\mathrm{pH}$ imaging. a
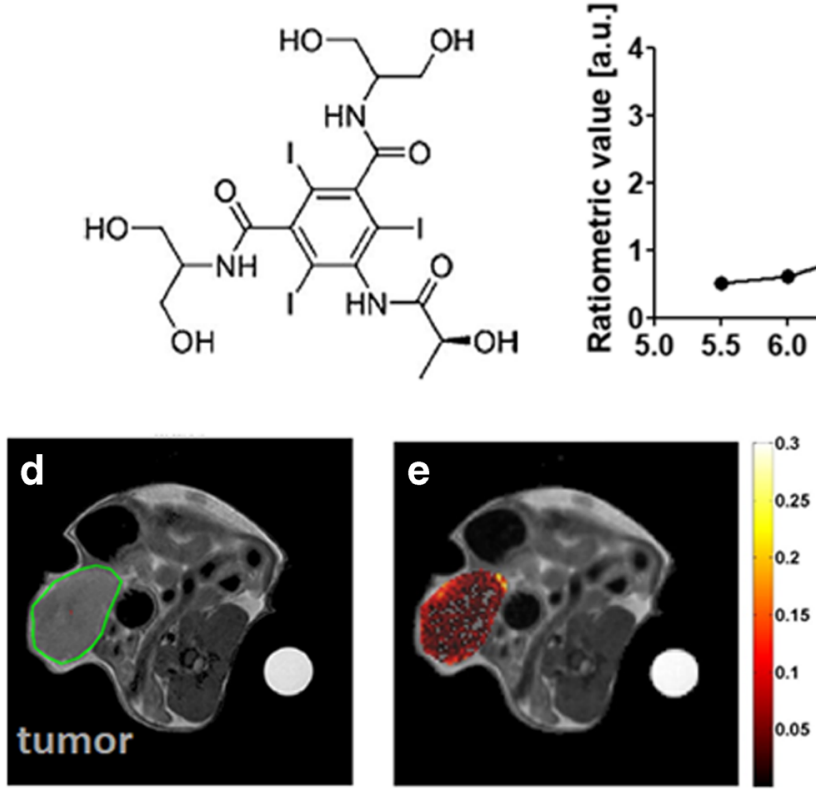

Fig. 4 MRI-CEST imaging of tumor $\mathrm{pH}$ using exogenous DIACEST probes. Chemical structure of Iopamidol (a), $\mathrm{pH}$ dependence of the ratiometric values (b) calculated by ratioing the ST\% contrast for the two amide groups (4.2 ppm and $5.5 \mathrm{ppm}$ ) and accuracy of the proposed method by comparing calculated vs. measured $\mathrm{pH}$ values (c) at $7 \mathrm{~T}$. Adapted with permission by John Wiley and Sons from Magnetic
C
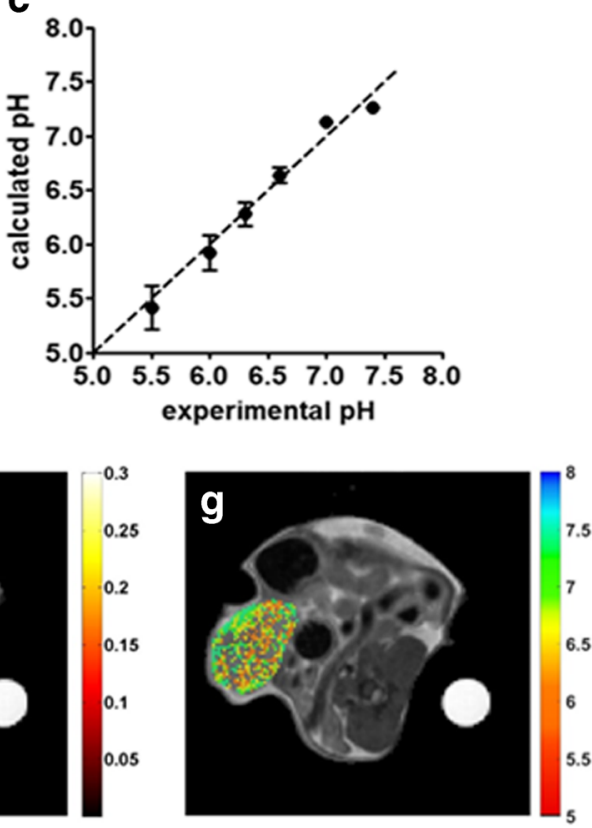

Resonance in Medicine 2011, 65, 202-211. Representative $T_{2 \mathrm{w}}$ anatomical images of a TS/A mammary bearing tumor mouse with tumor ROI highlighted in green (d). Contrast ST\% difference map calculated at $4.2 \mathrm{ppm}$ (e) and $5.5 \mathrm{ppm}$ (f) after Iopamidol i.v. injection (as ST\% post-ST\% pre injection) and corresponding calculated tumor $\mathrm{pHe}$ map over-imposed on the anatomical image (g) 

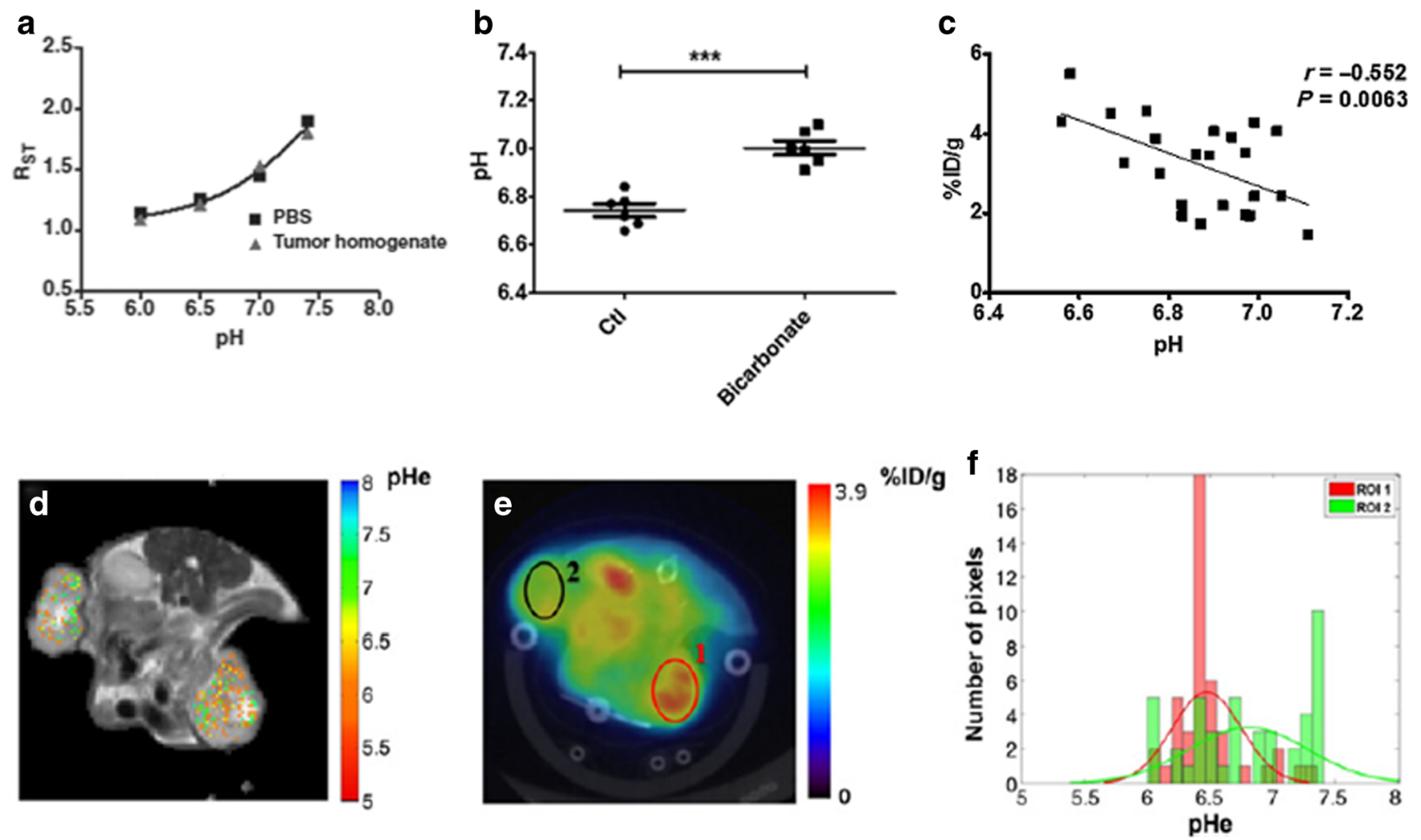

Fig. 5 First in vivo demonstration of the relationship between increased glucose uptake and tumor acidosis. Plot of the $\mathrm{pH}$ dependence of the ratiometric (RST) values in the $\mathrm{pH}$ range from 6 to 7.4 for Iopamidol in phosphate buffer solution and in tumor tissue homogenate at $3 \mathrm{~T}$ (a). Average tumor $\mathrm{pHe}$ values calculated in mice drinking natural or sodium bicarbonated water showing a statistically significant increase of tumor pHe after 5 days (b). Scatterplots with regression line (solid line) showing a marked and significant correlation between ${ }^{18} \mathrm{~F}-\mathrm{FDG}$ -

An analogous validation of DIACEST tumor $\mathrm{pH}$ mapping has been accomplished by Pagel's group, who used another $\mathrm{pH}$-sensitive X-ray contrast agent, Iopromide (Ultravist ${ }^{\circledR}$, Bayer Healthcare). Similarly to Iopamidol, the presence of two amide protons resonating at 4.2 and $5.6 \mathrm{ppm}$ allows a ratiometric approach for measuring $\mathrm{pH}$ in the range 6.6 to 7.2 with good $\mathrm{pH}$ precision [83]. Additional studies demonstrated that tumor $\mathrm{pHe}$ maps were correlated with biomarkers associated with tumor metabolism, showing tumor $\mathrm{pHe}$ differences between preclinical models of mammary carcinoma [84]. In vivo comparative studies between Iopamidol and Iopromide in two different tumor murine models of Raji lymphoma and of MCF-7 breast cancer xenograft revealed that Iopamidol can detect more precisely the tumor $\mathrm{pHe}$ [85] and that Iopamidol pHe mapping combined with ${ }^{18} \mathrm{~F}$-FDG PET can provide additional information for evaluating the therapeutic efficacy to metformin treatment in a pancreatic cancer model [86]. Since iodinated contrast media are approved for $\mathrm{CT}$ investigations, owing to the high safety profile, they have already been used in clinical trials for MRI-CEST $\mathrm{pH}$ mapping [87]. Of note, Iopamidol has been exploited for providing
PET \%ID/g uptake and MRI-CEST-derived tumor acidosis (c). Combined MRI $T_{2 \mathrm{w}} / \mathrm{CEST} \mathrm{pH}(\mathbf{d})$ and ${ }^{18} \mathrm{~F}$-FDG PET/CT (e) images of a representative TS/A tumor-bearing mouse. The tumor on the right side (ROI 1) shows a higher ${ }^{18} \mathrm{~F}$-FDG uptake in the PET image, corresponding to more acidic $\mathrm{pHe}$ values in the MRI-CEST $\mathrm{pHe}$ map, whereas tumor on the left side (ROI 2 ) is characterized by lower ${ }^{18} \mathrm{~F}$-FDG uptake values and corresponding less acidic $\mathrm{pH}$ values, as shown in the histogram plot (g). Adapted with permission from Cancer Research 2016, 76, 6463-6470

tumor pHe mapping in patients with ovarian or mammary cancers, showing, for the first time, clinical images of tumor acidosis (Fig. 7) [88]. Such important results are paving the way for further studies needed to confirm the diagnostic utility of tumor pHe mapping in the clinical scenario for both diagnostic and therapeutic monitoring.

In the above-reported ratiometric approach, there is the requirement of two resonances with different chemical shift for carrying out their selective irradiation. To extend the capability of DIACEST molecules to act as $\mathrm{pH}$ sensors, a novel ratiometric approach for molecules possessing only one mobile proton pool (hence potentially transforming every CEST compound in a $\mathrm{pH}$-sensitive agent) was developed. This approach has been validated by using another FDA-approved Xray agent, Iobitridol (Xenetix ${ }^{\circledR}$, Guerbet), with only one amide group resonating at $5.6 \mathrm{ppm}$ (Fig. 8). It was shown that irradiating the same mobile proton pool with different power level, the measured contrast curves (with distinct $\mathrm{pH}$ dependence) can be ratioed, still preserving a good $\mathrm{pH}$ response in the $\mathrm{pH}$ range from 6 to 7.4 [89]. In vivo validation was demonstrated in a murine breast tumor model, where the measured 
Fig. 6 MRI-CEST tumor $\mathrm{pH}$ assessment of treatment response to novel anticancer therapies. Tumor extracellular $\mathrm{pH}$ maps (measured following Iopamidol injection) for representative mice at baseline, 3 days and 15 days for dichloroacetate (DCA) treatment (b) showing increased reduction of tumor acidosis. Changes in mean tumor $\mathrm{pH}$ values (in comparison to baseline tumor $\mathrm{pH}$ values) between untreated and treated mice with DCA at different time points (c) and correlation with lactate level Adapted with permission from International Journal of Oncology 2017, 51, 498-506 control group (a) or upon measured in excised tumors (d).
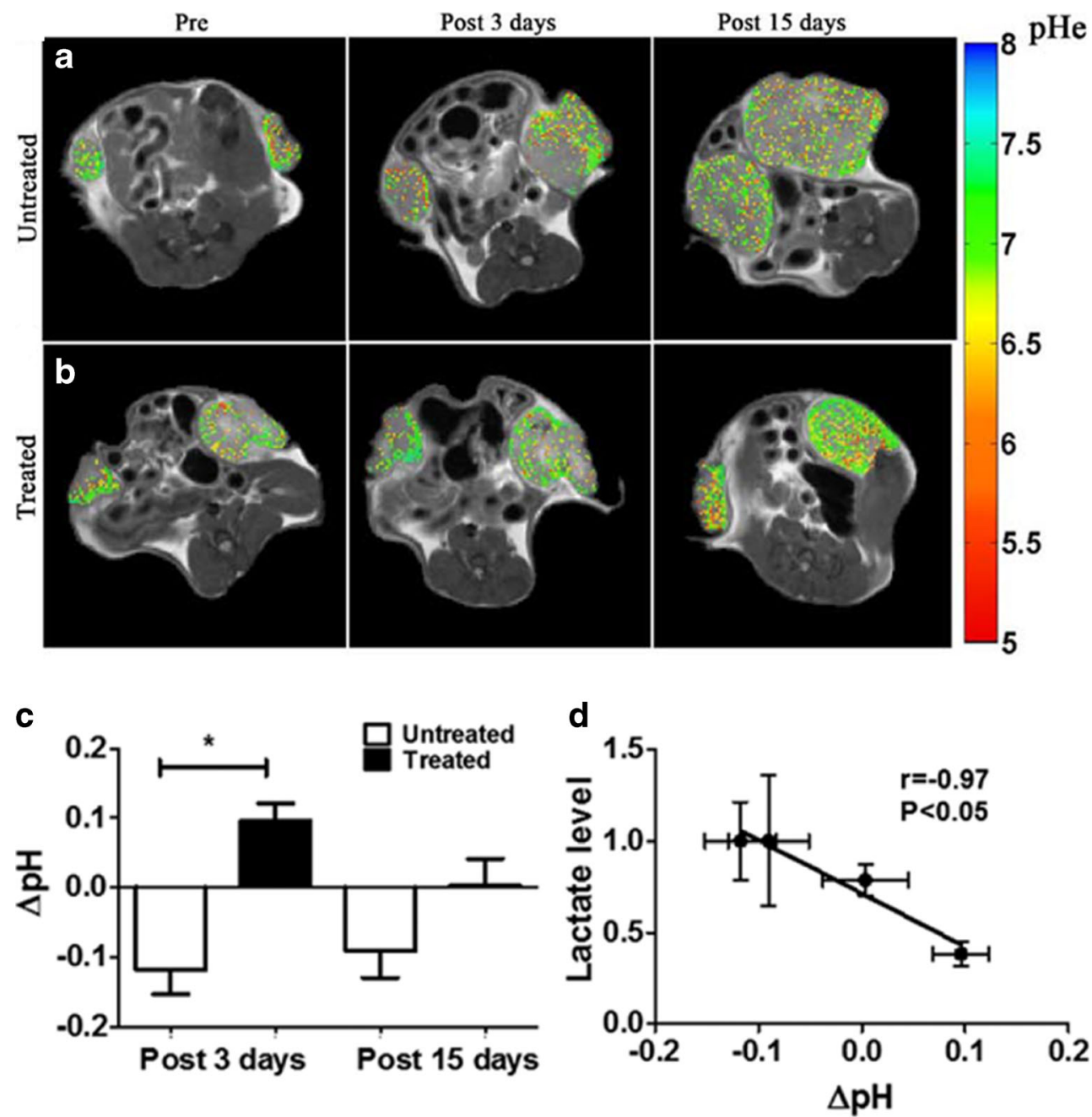

Fig. 7 MRI-CEST imaging of tumor $\mathrm{pH}$ at clinical level.

Representative patient with highgrade invasive ductal carcinoma and anatomical $T_{2}$-weighted image (a) and parametric iopamidol concentration map (b) and tumor $\mathrm{pHe}$ map calculated by Bloch fitting (c) or by Lorentzian fitting (d) procedures overlaid on the anatomical image, showing, for the first time, tumor acidosis in patients. Adapted by permission from Springer Nature, Molecular Imaging and Biology 2017, 19, 617-625 a

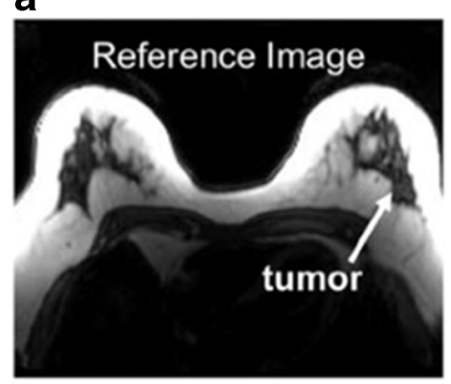

C

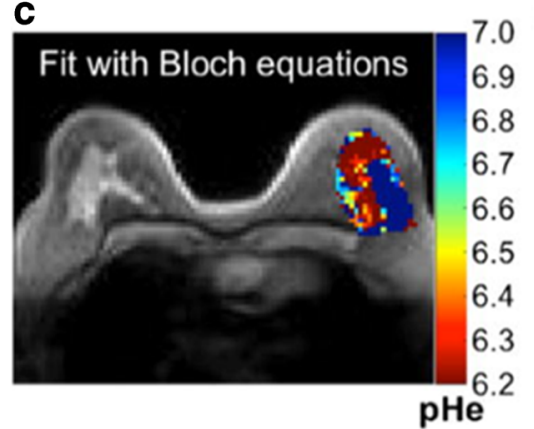

b

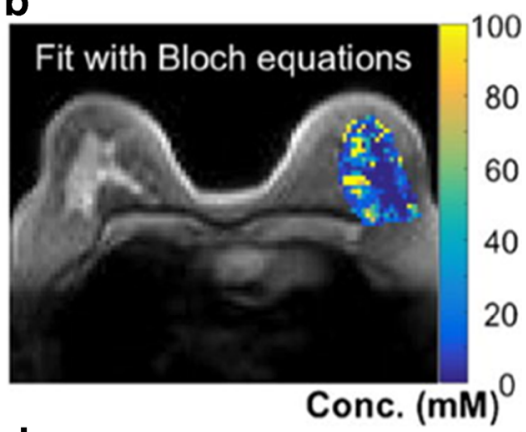

d

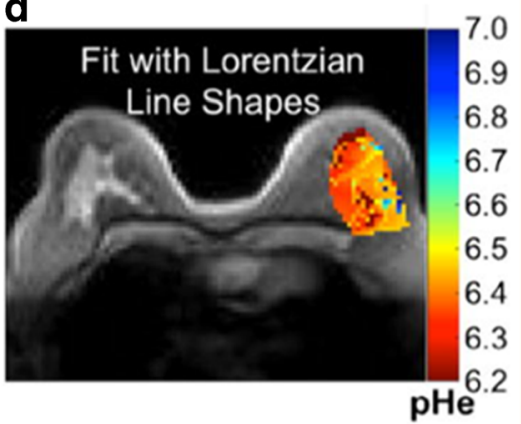


a<smiles>CN(CC(O)CO)C(=O)c1c(I)c(NC(=O)C(CO)CO)c(I)c(C(=O)N(C)CC(O)CO)c1I</smiles>

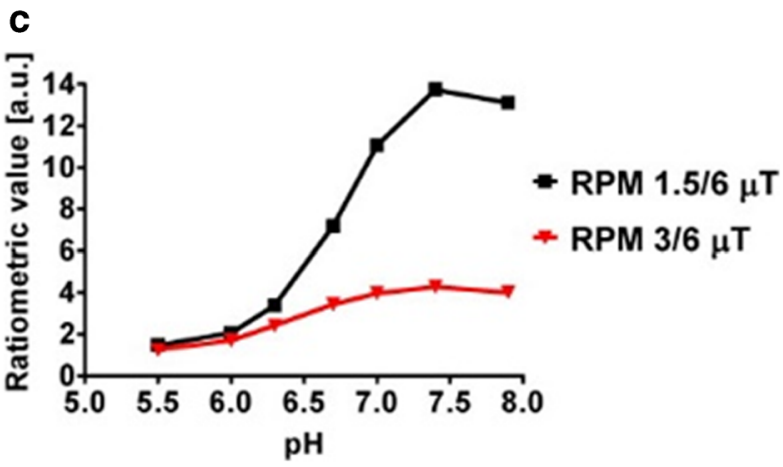

b

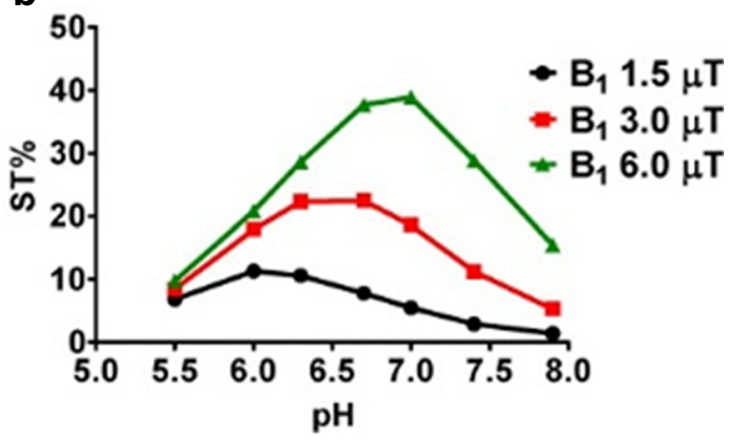

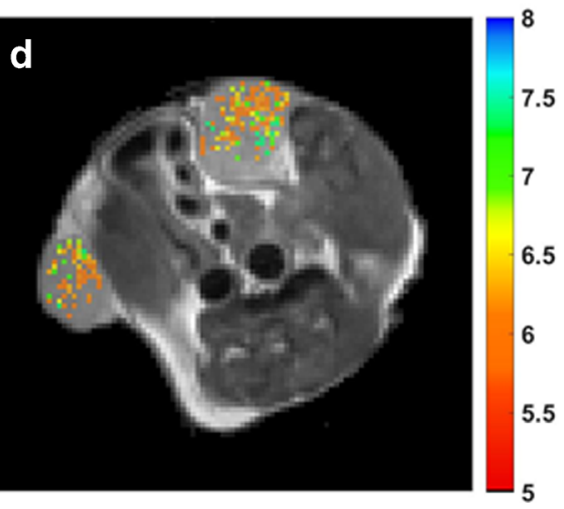

bearing mouse obtained upon rationing the difference ST map at $1.5 \mu \mathrm{T}$ and at $6 \mu \mathrm{T}$ after Iobitridol i.v. injection (d). Adapted with permission from Journal of the American Chemical Society 2014, 136, 14,33314,336, https://pubs.acs.org/doi/10.1021/ja5059313. Copyright 2014 American Chemical Society

cations) may display markedly increased chemical shift separations from the water resonance, which allow to access faster exchange rates before signal coalescence takes place [92]. The presence of mobile protons belonging to the coordinated water molecule or to chemical groups close to the coordinating metal cation has been exploited for generating the CEST contrast, which can be sensitized to a $\mathrm{pH}$-dependent exchange rate. CEST properties of lanthanide $(\mathrm{Ln}=\mathrm{Eu}, \mathrm{Dy}, \mathrm{Ho}, \mathrm{Er}, \mathrm{Tm}$, $\mathrm{Yb}$ ) complexes with the macrocyclic DOTAM-Gly ligand have been investigated for this purpose [93]. These complexes possess two groups of exchangeable protons at different chemical shifts: the $\mathrm{pH}$-sensitive amide protons as well as the directly metal-coordinated water protons. Among the investigated Ln-complexes, Yb-DOTAM-Gly showed the highest $\mathrm{pH}$ responsiveness in the $\mathrm{pH}$ range 5.5-8.1. However, to provide $\mathrm{pH}$ measurement independent from concentration, a mixture of two complexes, Eu-DOTAM-Gly and Yb-DOTAM-Gly need to be used to set up a ratiometric method, although this approach has not yet been validated in vivo. A solution to overcome the problem of the double (or cocktail) injection (i.e., the occurrence of different pharmacokinetics and biodistribution of two agents) was presented by Delli Castelli et al. who proposed a ratiometric approach based on ratioing the saturation transfer effects from the amide group ar at low magnetic fields. Paramagnetic CEST contrast agents (i.e., metal complexes of lanthanides or other metal 
( $\mathrm{pH}$ sensitive) and water ( $\mathrm{pH}$ independent) occurring in the same molecule [94]. Another solution was proposed with the Ytterbium $(\mathrm{Yb})$ lanthanide analogue of the clinical Gd-based HPDO3A (Prohance) contrast agent that was considered as a PARACEST $p H$-responsive agent [95]. Within this molecule, the presence of two equilibrium diastereoisomers of $\mathrm{Yb}^{3+}$ HPDO3A in solution results in two CEST signals arising from the same hydroxyl proton close to the paramagnetic center with different MR absorption frequencies (77 and $91 \mathrm{ppm}$, respectively), thus allowing a ratiometric approach (Fig. 9). $\mathrm{Yb}^{3+}$-HPDO3A showed a $\mathrm{pH}$ sensitivity in the range 6.58.0 at a field strength of $7 \mathrm{~T}$ and demonstrated in vivo tumor pHe maps in a murine melanoma model [96]. In a recent study using $\mathrm{Yb}^{3+}$-HPDO3A, it was shown that tumor pHe mapping can also provide information about tumor aggressiveness in a rat glioma model [97]. The advantage of PARACEST agents endowed with highly shifted resonances is further witnessed in the case of $\mathrm{Tm}^{3+}$-HPDO3A $\mathrm{pH}$-sensitive agent, which allows in vivo tumor $\mathrm{pH}$ mapping at lower magnetic fields ( $1 \mathrm{~T}$ ), due to the far-shifted hydroxyl resonances of the two diastereoisomers (71 and $185 \mathrm{ppm}$, respectively) [98]. PARACEST agents possessing two sets of exchangeable protons in the same structure were also investigated by Pagel's group who reported that, in $\mathrm{Yb}^{3+} \mathrm{DO} 3 \mathrm{~A}-\mathrm{oAA}$, a complex containing both amide and aryl amine protons, the formation of intramolecular hydrogen bond with a proximal carboxylate ligand resulted in a reduction of the amine exchange rate, thus generating a second CEST effect [99]. By combining high magnetic field and moderate power saturation levels, intratumoral injection of the paramagnetic agent was used to produce a parametric map of tumor $\mathrm{pHe}$.

Since PARACEST agents display resonances with large chemical shifts, the position of the resonating peak(s) can be exploited as an alternative approach for reporting on $\mathrm{pH}$ values. Sherry and coworkers developed a Europium-based contrast agent possessing a quite large shift in frequency of the metal-bound water molecule due to the delocalization of negative charge coming from the deprotonation of phenolic residue [100]. This process is driven by $\mathrm{pH}$ changes, where a change from 6.0 to 7.6 at $298 \mathrm{~K}$ leads to a shift for the water ${ }^{1} \mathrm{H}$-signal of 4 ppm, i.e., from 50.5 to 54.5 ppm respectively. By recording the CEST spectrum on a 9.4-T scanner, the measurement of the chemical shift of the absorbance peak provides direct estimates of tissue $\mathrm{pH}$ in a concentrationindependent manner without the need to set up a ratiometric approach [101]. Although promising, it has not yet been validated for measuring tumor pHe. Moreover, since PARACEST agents are sensitive to temperature changes, accurate maintenance of temperature homogeneity is necessary for a correct $\mathrm{pH}$ estimation.

As the PARACEST method detects proton exchange between bulk water and any exchangeable sites (on the ligand itself or the inner sphere of bound water) that are shifted by the paramagnetic $\mathrm{Ln}^{3+}$ ion, another approach has been exploited a

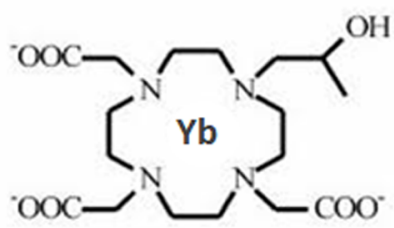

b

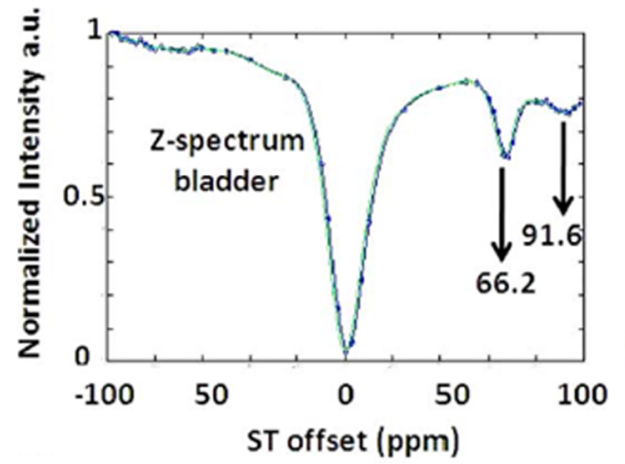

C

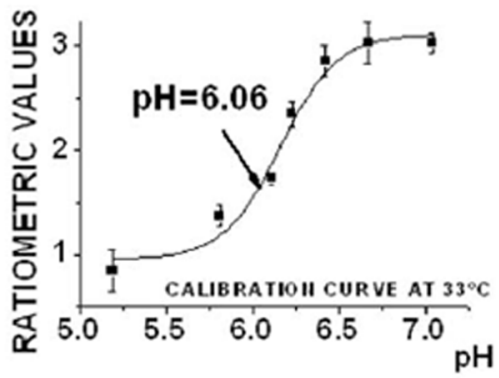

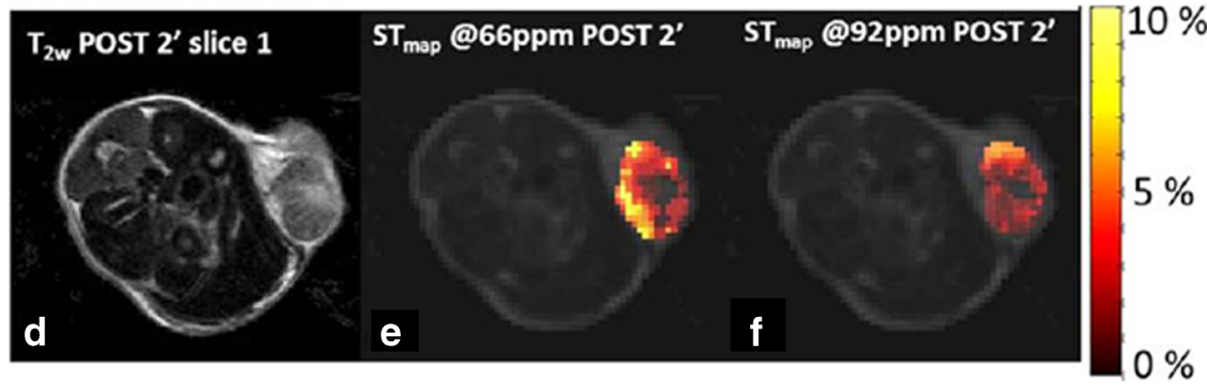

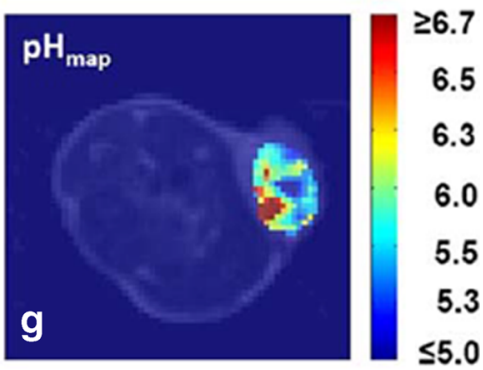

Fig. 9 MRI-CEST imaging of tumor $\mathrm{pH}$ using exogenous PARACEST probes. Chemical structure of Yb-HPDO3A (a), Z-spectrum from the bladder region 15 min after the i.v. injection of $\mathrm{Yb}$-HPDO3A (b) and $\mathrm{pH}$ calibration curve measured at $33{ }^{\circ} \mathrm{C}(\mathbf{c}) . T_{2 \mathrm{w}}$ image (d) and ST

maps calculated when the irradiation offset is set at $66 \mathrm{ppm}(\mathbf{e})$ and at $92 \mathrm{ppm}$ (f) post $2 \mathrm{~min}$ after the i.v. injection of Yb-HPDO3A and measured tumor $\mathrm{pH}$ map (g). Adapted with permission from Magnetic Resonance in Medicine 2014, 71, 326-332 
interrogating non-exchangeable protons, in line with the early report of Aime et al. [30]. This method, dubbed biosensor imaging of redundant deviation in shifts (BIRDS), utilizes shifts of non-exchangeable protons from macrocyclic chelates complexed with paramagnetic lanthanide ions to generate $\mathrm{pHe}$ maps [102, 103]. The most recent application of the BIRD approach used $\mathrm{TmDOTP}^{-}$to investigate tumor $\mathrm{pHe}$ differences in two glioma rat models, showing more acidic peritumoral pHe values in the more aggressive tumors [104]. A major concern regarding this approach was that renal ligation was needed to stop the renal clearance of the agent in order to allow a higher accumulation inside the tumor and, consequently, get higher sensitivity. In addition, very high magnetic fields $(11.7 \mathrm{~T})$ were needed to finely resolve small shifts in the resonances, limiting the attainable $\mathrm{pH}$ accuracy.

As a final remark on PARACEST agents, one may note that they are more sensitive than DIACEST agents as they are not influenced by the water-indirect saturation or by concomitant effects arising from the endogenous semisolid protein-based components. However, high doses are still required to reach sufficient concentrations at the region of interest for attaining detectable CEST signals, as well as high power levels are required for an efficient saturation of the fast-exchanging protons, which is not readily available on clinical scanners.

\subsection{MRI—spin-lock}

Besides the slow/intermediate exchange regime that is the basis of CEST, fast chemical exchange of mobile protons with water can be exploited for generating contrast in MR images, using a method based on the "spin-lock" (SL) technique [105]. With this approach, the water magnetization is first flipped from the $Z$-axis and then locked by radiofrequency pulses. During the applied SL pulse, the longitudinal water magnetization decay in the rotating frame $\left(T_{1 \rho}\right)$ is sensitive to chemical exchange contributions, therefore providing an alternative method for exploring chemical exchange-based contrast [106]. In this context, molecules possessing fastexchanging hydroxyl protons such as glucose can be detected more easily with the SL than with CEST $[107,108]$. Iodinated $\mathrm{X}$-ray agents possessing hydroxyl groups have also been investigated by the SL approach by Gore and coworkers [109]. The same group has exploited Iohexol (Omnipaque®, GE Healthcare), a CT contrast medium for mapping rat brain tumor pHe by exploiting the spin-lock approach at very high magnetic field (9.4 T) [110]. By measuring the differences in the $T_{1 \rho}$ dispersion profiles (i.e., a measure of $T_{1 \rho}$ values as a function of the power of the locking field) before and after the injection, and then fitting this $\Delta T_{1 \rho}$ dispersion difference, it was possible to quantify the characteristics of the exchanging proton pools. According to a measured calibration curve, the tumor $\mathrm{pHe}$ values correspond to an extracellular $\mathrm{pH}$ ranging from 6.6 to 7.0 (Fig. 10). Compared with CEST imaging of X- ray contrast, the SL technique requires higher powers and longer acquisition times to improve image quality, but translation to clinical scanners may be feasible.

\subsection{MRI-hyperpolarized}

Dynamic nuclear polarization (DNP) transfers polarization from electrons to nuclei, which provides a dramatic increase of sensitivity in MRS, particularly for low- $\gamma$ nuclei, such as ${ }^{13} \mathrm{C}$ [111]. A mix of ${ }^{13} \mathrm{C}$-labeled molecules with small quantities of a stable free radical cooled to $\sim 1 \mathrm{~K}$ in a magnetic field and continuously irradiated at the electron paramagnetic resonance (EPR) absorption frequency of the radical species. This results in the transfer of polarization from the unpaired electron to the ${ }^{13} \mathrm{C}$ nuclei [112]. Because the resonance frequencies of electrons are thousands of times higher than those of NMR nuclei, this transfer dramatically increases the polarization of the NMR nuclei, directly increasing the sensitivity theoretically up to 50,000-fold. In practice, increases in the signal-to-noise ratio of 10,000 are routinely achieved for DNP-hyperpolarized ${ }^{13} \mathrm{C}$-labeled molecules, allowing their direct detection with spectroscopic imaging, MRSI [113, 114]. The development of hyperpolarized molecules whose chemical shift is $\mathrm{pH}$ dependent can provide another method for in vivo $\mathrm{pH}$ imaging with high sensitivity.

Brindle et al. firstly described the injection of hyperpolarized ${ }^{13} \mathrm{C}$-bicarbonate as a $\mathrm{pH}$-responsive agent, exploiting the acid-base equilibrium between $\mathrm{H}^{13} \mathrm{CO}_{3}{ }^{-}$and ${ }^{13} \mathrm{CO}_{2}$ that show distinct chemical shifts. According to the Henderson-Hasselbalch equation, the ratio of $\mathrm{H}^{13} \mathrm{CO}_{3}{ }^{-}$and ${ }^{13} \mathrm{CO}_{2}$ resonance intensities can be exploited for assessing $\mathrm{pH}$. The method has been tested for mapping the extracellular $\mathrm{pH}$ in a lymphoma tumor murine model [115]. The $\mathrm{pH}$ map calculated after the intravenous injection of $100 \mathrm{mM}$ hyperpolarized ${ }^{13} \mathrm{C}$-bicarbonate gave an average calculated $\mathrm{pH}$ of $6.71 \pm 0.14$. Scholl and coworkers applied this technique for studying temporal changes of tumor $\mathrm{pHi}$ and $\mathrm{pHe}$ during tumor growth in a rat glioma model [116]. Despite ${ }^{13} \mathrm{C}$ labeled bicarbonate has been initially proposed as a probe for tumor pHe imaging, the transport of $\mathrm{CO}_{2}$ and bicarbonate in and out the cells results in a mixed contribution of intra- and extracellular values to the measured $\mathrm{pH}$.

Recently, a new $\mathrm{pH}$-responsive probe has been introduced based on the chemical structure of the $\left[1,5-{ }^{13} \mathrm{C}_{2}\right]$ zymonic acid (ZA) [117]. ZA was identified as an impurity within ${ }^{13} \mathrm{C}$ pyruvic acid and it was subsequently shown to act as a $\mathrm{pH}$ sensitive molecule. The in vivo application described by Schwaiger, Schilling et al. of this new probe in mammary MAT B III tumors showed the detection of extravascular acid compartment by ZA signal that was in good agreement with three other independent $\mathrm{pH}$ measurements, suggesting the ability of ZA to measure and discriminate between extravascular $\mathrm{pH}$ and intravascular $\mathrm{pH}$. The accuracy of $\mathrm{pH}$ 
a
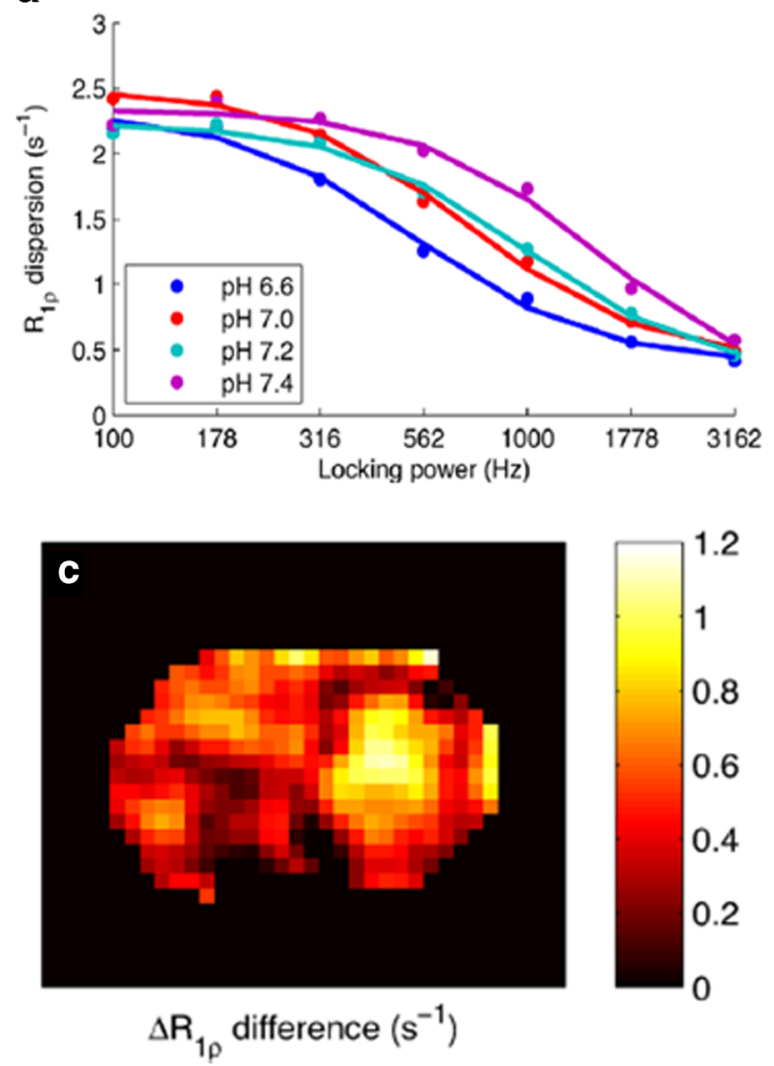

Fig. 10 MRI-spin-lock imaging of tumor $\mathrm{pH}$. Measured $R_{1 \rho}$ dispersion curves for Iohexol at several $\mathrm{pH}$ values but constant agent concentration (a) and calibration curve for the fitted $\mathrm{s} 0$ parameter as a function of $\mathrm{pH}$ (b). $\Delta R_{1 \rho}$ difference map at $10 \mathrm{~min}$ after injection (c) and corresponding

measurement ( $0.1 \mathrm{pH}$ unit) as well as the long signal lifetime ( $T_{1}$ of $17 \mathrm{~s}$ at $7 \mathrm{~T}$ in vivo) makes this new reporter valuable for further preclinical and clinical studies. Further, perdeuteration of ZA increased the $\mathrm{T} 1$ relaxation time significantly, prolonging hyperpolarization and increasing sensitivity [118], allowing dynamic monitoring of $\mathrm{pH}$ changes in vivo (Fig. 11). Likely, the development of novel MRI sequences for fast image acquisition and the improvement in signal detection by optimal design of the receiver coils will allow this technique to reach the sub-millimeter spatial resolution in the near future for high-resolution $\mathrm{pH}$ imaging. Unfortunately, the requirements of dedicated ${ }^{13} \mathrm{C}$ coils and the technical challenges for producing, via DNP, hyperpolarized molecules still hamper the clinical adoption of this promising technique. Other routes for generating hyperpolarized molecules are currently under investigation as they may allow more versatile approaches to the field $[119,120]$.

\section{Electron paramagnetic resonance imaging}

Electron paramagnetic resonance (EPR) is a spectroscopic technique based on the detection of unpaired electrons

\section{b}
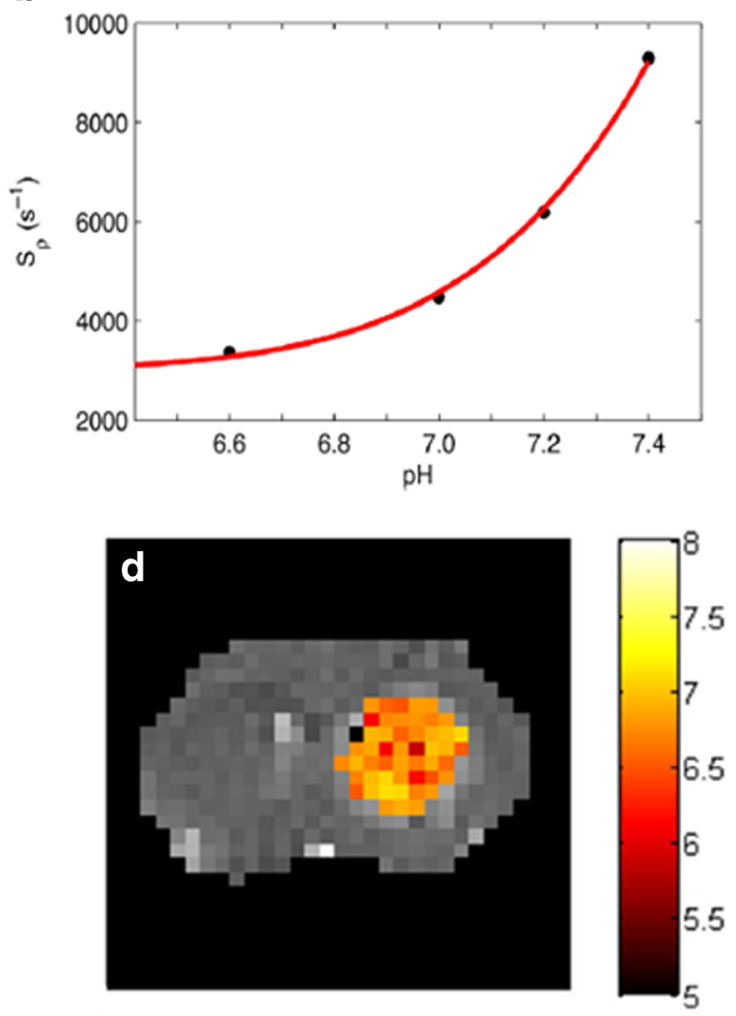

$\mathrm{pH}$ map from the tumor-bearing rat brain calculated as average of six $\Delta R_{1 \rho}$ difference maps at different time points after iohexol i.v. injection (d). Adapted with permission from Magnetic Resonance in Medicine 2018, 79, 298-305

(radicals) in paramagnetic species, exposing the sample to a sweep of microwave frequency irradiation. Because of the insufficient amount of radical species in viable tissues combined with their fast relaxation, the use of paramagnetic probes to reach a sufficient concentration at the site of interest is required [121]. Whereas direct detection of paramagnetic probes guarantees high specificity, development of probes endowed with good biocompatibility, long stability during the measurement, low toxicity, and optimal spectral sensitivity is not a straightforward task. Moreover, unlike MRI measurements, EPR experiments are performed in a continuous wave mode, resulting in long acquisition times, small sample size, and restricted to surface tissues. EPR imaging (EPRI) is challenging for anatomic co-localization since, at low magnetic field, the small polarization of the ${ }^{1} \mathrm{H}$ spin is not sufficient to generate MR images. Two approaches have been developed for imaging purposes, namely (i) the use of pulsed irradiations (pulsed-EPRI), which shortens the acquisition time [122], but still lacking of anatomical information, and (ii) the exploitation of the Overhauser effect. This technique, called OMRI (Overhauser enhanced magnetic resonance imaging), also known as proton electron double resonance imaging (PEDRI) was developed by Lurie et al. in 1988 [123]. In this 
Fig. 11 MRI-hyperpolarized imaging of tumor $\mathrm{pH}$. Chemical structure of $\left[1,5-{ }^{13} \mathrm{C}_{2}, 3,6,6,6-\mathrm{D}_{4}\right]$ zymonic acid $\left(\mathrm{ZA}_{\mathrm{d}}\right)$ with $T_{1}$ values and $\mathrm{pH}$-dependent chemical shifts of the $\mathrm{C} 1$ and $\mathrm{C} 5$ carbon atoms (a). In vivo signal intensity images acquired after tail-vein injection of ZA (b) and $\mathrm{ZA}_{\mathrm{d}}(\mathbf{c})$ overlaid on anatomical $T_{1}$-weighted ${ }^{1} \mathrm{H}$ images. $\mathrm{pH}$ images calculated from the chemical shift differences of $\mathrm{C} 5$ and urea, weighted by the respective signal intensities, show an acidification of the tumor microenvironment for both ZA (d) and $\mathrm{ZA}_{\mathrm{d}}(\mathbf{e})$. Reproduced with permission from ChemPhysChem 2017, 18, 2422-2425
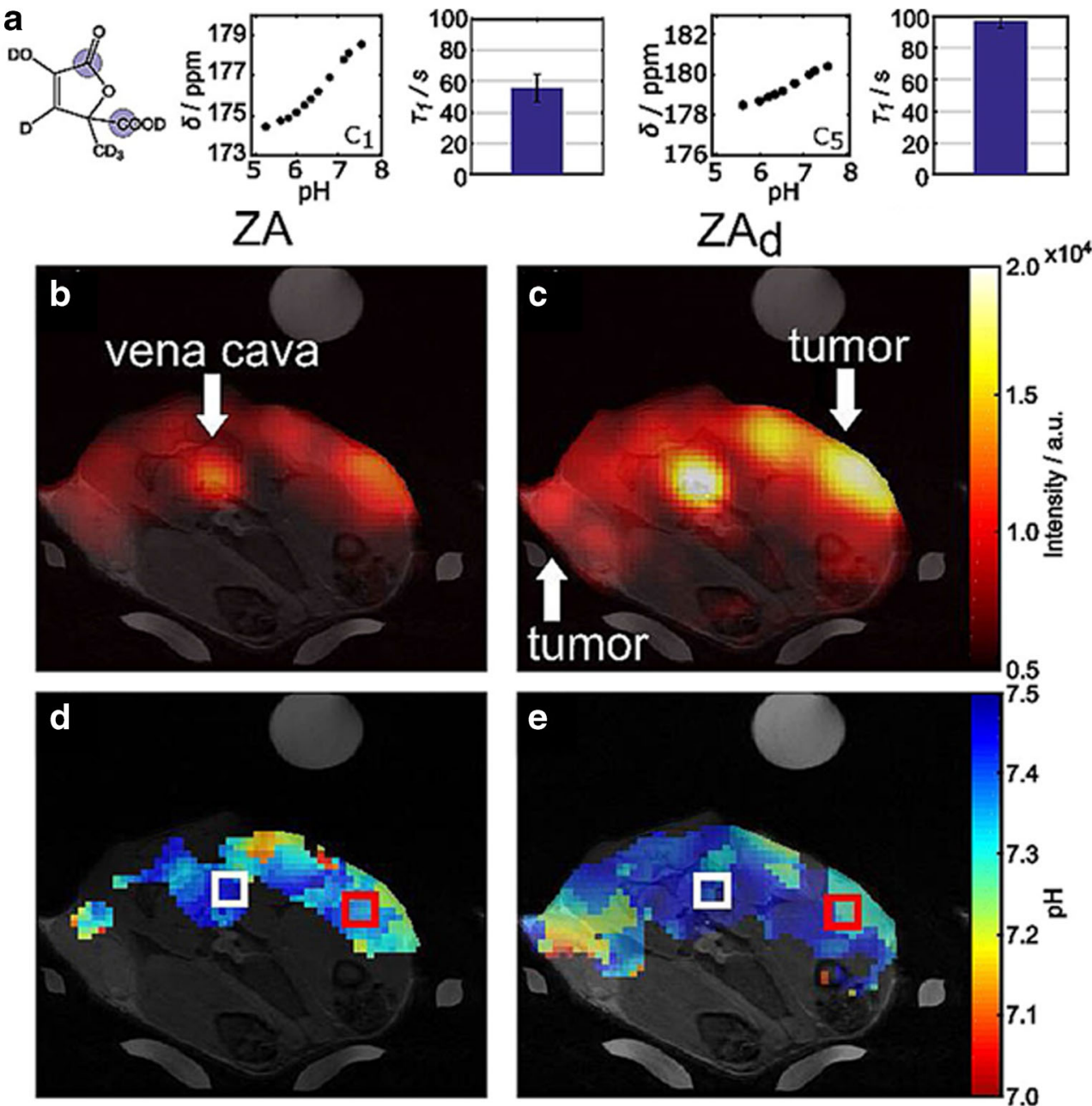

case, the electron spin polarization is transferred to ${ }^{1} \mathrm{H}$ polarization with subsequent MR image acquisition, resulting in an indirect detection of the radical probe [124]. Unlike EPRI, higher resolution can be achieved.

Khramtsov and coworkers have designed a $\mathrm{pH}$-sensitive nitroxide $\mathrm{R}_{1}$ and its deutero-substituted analog, $\mathrm{R}_{2}$, for EPR monitoring of $\mathrm{pHe}$ in breast cancer models [125]. The enhanced stability of the proposed probe conjugated with its extracellular localization and $\mathrm{pH}$ sensitivity in the range of 6.5-7.5 allowed ready detection of pHe. The same group investigated a dual probe pTAM (deuterated derivative of the phosphonated triaryl methyl or TAM) for simultaneous $\mathrm{pH}$ and oxygen monitoring thanks to the extraordinary stability toward tissue redox processes, longer relaxation time, and narrower line width of this probe compared to nitroxidebased probes [126]. Suffering of the lack of EPR spectral information, the conventional PEDRI approach was recently improved with the variable field and variable radio frequency (VRF) PEDRI, where the $\mathrm{pH}$ map was obtained from two PEDRI acquisitions performed at the EPR frequencies of protonated and unprotonated form of a deuterated $\mathrm{pH}$-sensitive probe, Im6, in Met-1 tumor-bearing mice [127]. The VRF
PEDRI technique was applied to obtain the $\mathrm{pH}$ map distribution in tumor with an average value of extracellular $\mathrm{pH}$ of 6.8 \pm 0.1 in agreement with the averaged $\mathrm{pH}$ values measured with the $\mathrm{pH}$ microelectrode.

More recently, Komarov and coworkers implemented three-dimensional (3D) EPR pH mapping in animal models by applying fast projection scanning with a constant magnetic field. Both dedicated instrumentations and complex reconstruction algorithms are needed for deriving $\mathrm{pH}$ images from the measured EPR spectra, following the administration of a pH-sensitive nitroxyl radical probe (dR-SG) [128]. This newly proposed EPR method was demonstrated in mapping $\mathrm{pH}$ of tumor progression in a murine squamous cell carcinoma model (Fig. 12).

EPR approaches are promising in offering accurate $\mathrm{pH}$ measurements and multiple functional information, beside tumor $\mathrm{pH}$, simultaneously. However, several drawbacks, including low spatial resolution $\left(2-3 \mathrm{~mm}^{3}\right.$ voxels, comparable to MRS), possible toxicity of the radical probes at the high required doses, demanding technical requirements, and high power radiofrequency irradiations, need to be solved before a large adoption of this approach can be foreseen. 
a

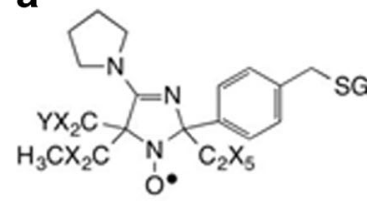

R-SG: $X=\mathrm{H} ; \mathrm{Y}=\mathrm{CH}_{3}$

dR-SG: $X=D ; Y=\mathrm{CH}_{3}(1 / 3) ; \mathrm{CD}_{3}(2 / 3)$

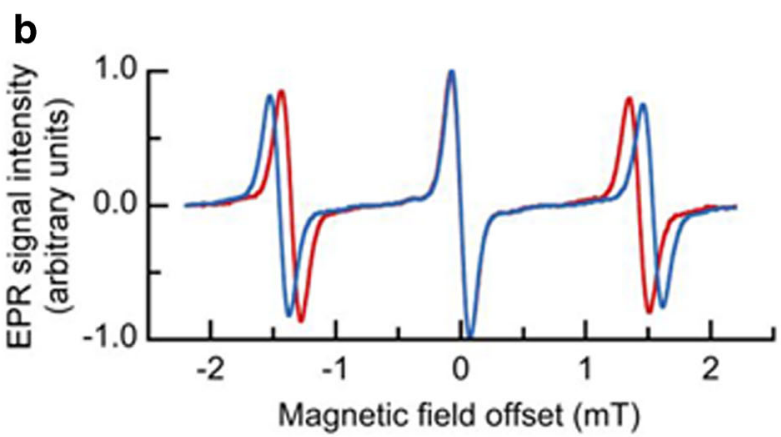

Day 5
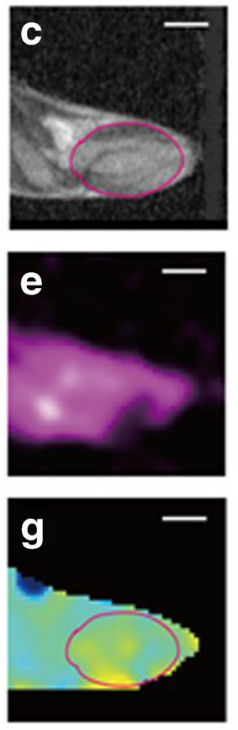

Day 8
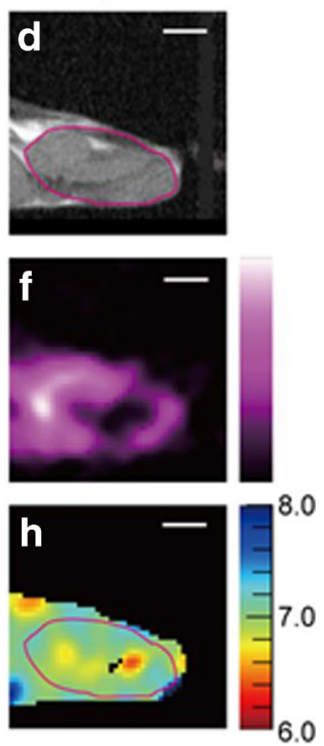

Fig. 12 EPR-based imaging of tumor $\mathrm{pH}$. Chemical structures and scheme of protonation of the $\mathrm{pH}$-sensitive nitroxyl radical (R-SG) and its deuterium-enriched (dR-SG) analog (a). First derivative EPR spectra of $2 \mathrm{mM} \mathrm{dR}-\mathrm{SG}$ measured at $750 \mathrm{MHz}$ in alkaline ( $\mathrm{pH}=10.0$, blue line) and acidic $\left(\mathrm{pH}=3.0\right.$, red line) solutions (b). $T_{2}$-weighted proton MR anatomical images of the SCC VII tumor-bearing mouse leg in the

\section{Positron emission tomography imaging}

Positron emission tomography (PET) imaging is widely used in both preclinical and clinical imaging. It is characterized by a high sensitivity and limitless penetration depth and allows absolute quantification of the concentration of the injected tracer. However, PET is characterized by a low spatial resolution $\left(\sim 2 \mathrm{~mm}^{3}\right)$ that limits its application for evaluation of heterogeneity in tumors [129]. Until now, only few $\mathrm{pH}-$ sensitive probes have been designed and tested, but have not yet been able to provide quantitative tumor $\mathrm{pH}$ images. Indeed, these probes can report on the presence of acidic extracellular environment only on the basis of their preferential accumulation, instead of providing a readout of the actual $\mathrm{pH}$ values. The first of these $\mathrm{pH}$-responsive probe was a peptide (pHLIP) that changes its conformation in a $\mathrm{pH}$-dependent manner (from random coil to alpha-helix), promoting its penetration into the cellular membranes at low extracellular $\mathrm{pH}$ values (Fig. 13) [130]. In vitro tests showed that at $\mathrm{pH} 7.4$ less than $5 \%$ of the compound is incorporated into the membrane, while at $\mathrm{pH} 6$, this value is more than $25 \%$. In a preliminary study, ${ }^{64} \mathrm{Cu}$-DOTA-pHLIP was successfully imaged in prostate tumors (LNCaP and PC-3) showing a higher uptake and retention in the LNCaP tumors, whereas the uptake was reduced in mice that received 7 days of bicarbonated water [131]. In another study, pHLIP was bound to two positron emitting radionuclides $\left({ }^{64} \mathrm{Cu}\right.$ and $\left.{ }^{18} \mathrm{~F}\right)$ and tested in breast (4T1), prostatic (LNCaP and PC3), and melanoma (B16f10) tumor models [132]. ${ }^{64} \mathrm{Cu}$ has a moderately short half- sagittal plane, acquired at day $5(\mathbf{c})$ and $8(\mathbf{d})$, respectively, and representative images of EPR signal intensity reconstructed from the $3 \mathrm{D}$ data (e, f) and corresponding tumor $\mathrm{pHe}$ maps at the two time points $(\mathbf{g}, \mathbf{h})$. The white scale bar on the images corresponds to $5 \mathrm{~mm}$. Adapted with permission from Analytical Chemistry 2018, 90, 13,93813,945

life and high spatial resolution that is an advantage for clinical application; however, its production is complicated and expensive. Conversely, ${ }^{18} \mathrm{~F}$ has longer half-life but it is routinely produced at numerous sites worldwide. In the slower growing tumors (PC3 and LNCaP), the tumor environment is less acidic, resulting in a lower accumulation; conversely, in more acidic and faster growing 4T1 and B16-F10 tumors, the accumulation was higher. Alternatively to pHLIP, Flavell et al. proposed an acid-labile precursor imaging tracer based on ${ }^{18} \mathrm{~F}-\mathrm{FDG}$ with a glycosylamine linkage. In an acidic environment, the probe decomposes to release the ${ }^{18} \mathrm{~F}$-FDG that accumulates in the cancerous cells. The probe was tested in a PC3 xenograft tumor model, showing a similar tumor uptake when compared to ${ }^{18}$ F-FDG, but capable of distinguishing changes in tumor acidosis following bicarbonate treatment [133]. This new class of PET tracer represents a promising strategy for addressing tumor acidosis, with the advantage of using the ${ }^{18} \mathrm{~F}$-FDG derivatives at tracer doses. However, the accumulation of this tracer is still dependent on the presence of GLUT transporters, therefore reducing the exploitation in less glucose avid tumors, as for ${ }^{18}$ F-FDG. Overall, the PETbased approaches can only provide qualitative information of tumor acidosis with limited spatial resolution.

\section{Optical imaging}

Optical imaging is characterized by high in-plane resolution and sensitivity, and excellent sensitivity and temporal 
a

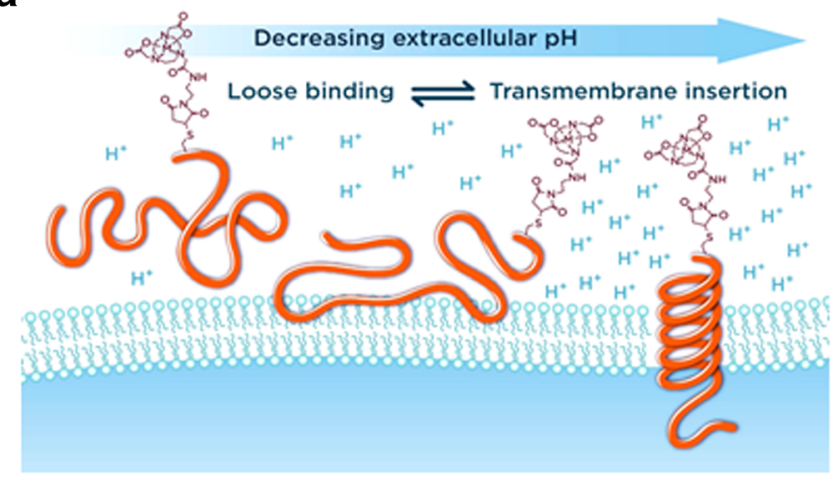

C

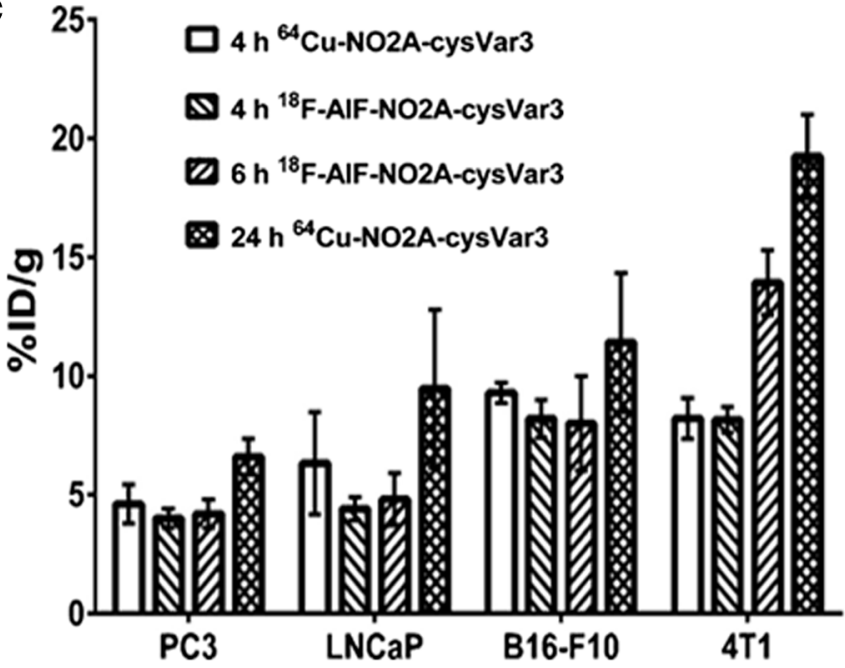

b

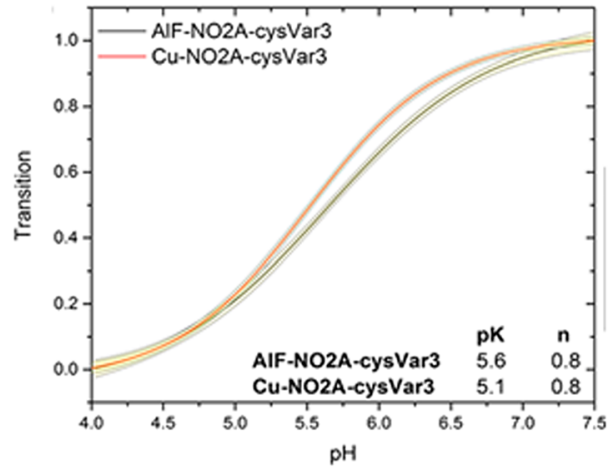

d

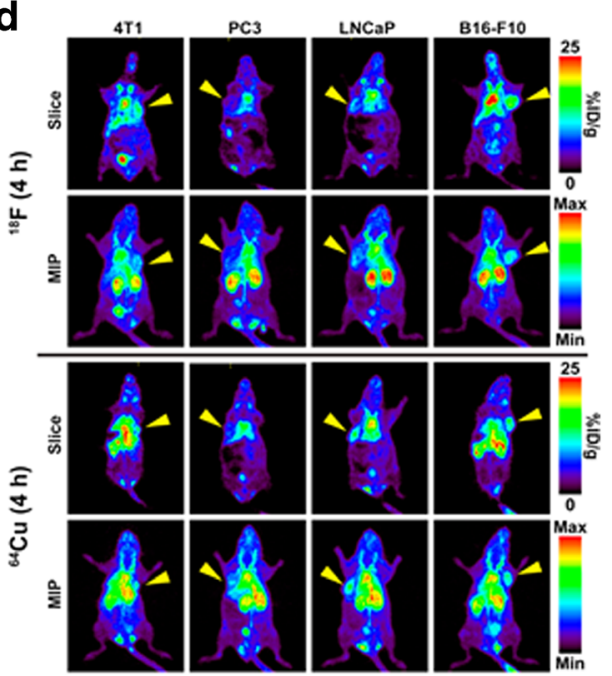

Fig. 13 PET-based imaging of tumor $\mathrm{pH}$. Schematic cartoon of the different folding states of $\mathrm{pHLIP}$ peptide in decreasing extracellular $\mathrm{pH}$ conditions (a). pH-dependent bilayer insertion of NO2A-cysVar3 constructs (b) and graph (c) of the ex vivo tumor uptake (\% $\mathrm{ID} / \mathrm{g})$ for different radiolabeled peptides $\left({ }^{64} \mathrm{Cu}\right.$ and $\left.{ }^{18} \mathrm{~F}\right)$ in several tumor models (prostate: PC3 and LNCaP, melanoma: B16F10, and breast: 4T1) and

resolution. However, it is limited in depth penetration and quantification, due to the well-known issues of absorption, reflection, and refraction processes when the emitted photons travel within biological tissues [134]. Importantly, in the NIR range (650-900 nm), photons travel through tissue deeper than photons in the visible range, and this wavelength range, called in vivo optical window, has been extensively exploited for addressing biological questions following the development of fluorescent-responsive probes [135].

SNARF-1 (semi-napthorhodafluorescein-1) is a fluorescent molecule that exhibits a $\mathrm{pH}$-dependent emission spectral shift from 580 to $640 \mathrm{~nm}$. The ratio of the measured fluorescent signals between these two wavelengths allows accurate $\mathrm{pH}$ measurements. Since the excitation and emission wavelengths are in the visible range, this probe can be applied in cell cultures or in superficial tumors like in rabbit ear chambers [136] or in intravital microscopy with the support of a window chamber [8]. To date, several studies using the window chamber tumor model have addressed important time points $(4,6$, and $24 \mathrm{~h})$. Slices and maximum intensity projections (MIP) PET images of radiolabeled peptides in tumor models at $4 \mathrm{~h} \mathrm{(d).}$ The yellow arrowheads indicate where the tumor is located in each mouse. Adapted with permission from Bioconjugate Chemistry 2016, 27, 2014-2023

questions regarding the role of tumor acidosis in driving tumor invasion. In particular, by using this probe, Gillies and coworkers demonstrated that regions of highest tumor invasion correspond to areas of lowest $\mathrm{pH}$ [9]. On this basis, the administration of sodium bicarbonate to reduce tumor acidity (as demonstrated by the increase of the measured tumor $\mathrm{pH}$ values by this approach) was proposed as a novel therapeutic strategy to reduce tumor metastases [137]. Although the dorsal skinfold window chamber is commonly used to evaluate the microvasculature in various settings in vivo, it has to be stressed that a surgical operation is needed for implanting the window chamber and this operation could influence angiogenic processes. Moreover, $\mathrm{pH}$ measurements via SNARF-1 can be affected by difference in temperature between the calibration curve and the imaged tissue, as well as upon interaction of the fluorescent probe with extracellular proteins that can affect the $\mathrm{pH}$ dependence of the fluorescent spectra [138].

To measure in vivo tumor $\mathrm{pH}$ without the support of a window chamber, different probes with excitation and 
emission wavelength in the NIR region have been developed. Most of these approaches require two fluorescent probes, one $\mathrm{pH}$ sensitive and one $\mathrm{pH}$ insensitive, to set up a ratiometric approach for measuring $\mathrm{pH}$ values independently from the probes' concentration. The conjugation of the two fluorescent dyes to a nanosized carrier guarantees the same concentration of the two molecules, solving the limitations associated with the assumption of the similar pharmacokinetic and biodistribution. However, the increase in the size of the resulting adducts, according to the final size and charge of the nanosystem, may limit the extravasation in poor perfused tumors or promote macrophage uptake and reduce the elimination rate, raising obvious toxicity issues. As an example, a $\mathrm{pH}$-sensitive cyanine dye (CypHer5E), and a $\mathrm{pH}$-insensitive fluorescent dye (Oyster800) have been conjugated on the surface of a biocompatible sphere with a diameter of $100 \mathrm{~nm}$. CypHer5E has minimal fluorescence at neutral $\mathrm{pH}$ but becomes highly fluorescent with an emission peak at about $670 \mathrm{~nm}$ in an acidic environment. By taking the ratio of fluorescence intensities at different wavelengths, it was possible to detect tumor $\mathrm{pH}$ changes during tumor progression in a melanoma (B16f10) tumor murine model [139].

Another $\mathrm{pH}$-ratiometric approach can be set up by using fluorescent dyes displaying two absorption peaks, one $\mathrm{pH}$ independent and the second $\mathrm{pH}$ dependent, respectively. The ratio of the fluorescent emission signals following the excitation at these two peaks makes the $\mathrm{pH}$ measurement independent of concentration. Besides the SNARF-1 pH sensor discussed above, rhodamine molecules were also proposed, with promising properties thanks to strong resistance to photobleaching, high water solubility, and high fluorescence quantum yields for both the protonated and deprotonated forms. Recently, a modified rhodamine bound to dextran (Dex-Me-pEPPR) has been synthetized and tested as a ratiometric NIR $\mathrm{pH}$-sensitive probe. The intravenous administration of Dex-Me-pEPPR showed a more acidic value in the tumor region compared to healthy region (Fig. 14), in good agreement with the $\mathrm{pH}$ values measured using a $\mathrm{pH}$ electrode [140].

Finally, a different class of NIR pH-sensitive probes composed of systems that are activated by acidic $\mathrm{pH}$ has been proposed. For example, Gao and coworkers synthetized a 30-nm nanoparticle made of a $\mathrm{pH}$-sensitive copolymer and loaded with the Cy5.5 dye. At blood $\mathrm{pH}$ values (7.4), the fluorescence is quenched, but at acidic $\mathrm{pH}$ values (6.9), the micelles dissociate, resulting in a marked increase of the fluorescence signal that allows detection of the acidic microenvironment of tumors [141]. A similar approach has been adopted to detect tumor acidosis by exploiting two NIR fluorophores (IR783) conjugated via a flexible and acid liable linkage. At neutral $\mathrm{pH}$ conditions, the fluorescence of this probe is quenched due to intramolecular dimeric aggregate.
Fig. 14 Optical imaging of tumor $\mathrm{pH}$. Chemical structure of phosphorus-substituted rhodamine-based probe (a). $\mathrm{pH}$ calibration curves of several substituted rhodamine probes based on the ratio of fluorescence intensity at $650 \mathrm{~nm}$ and $705 \mathrm{~nm}$ with $\mathrm{pK}$ a values of the probes given in parentheses (b). In vivo fluorescence ratio images of mice with subcutaneous tumors (c, $\mathrm{K}$ kidney, $\mathrm{N}$ normal tissue, $\mathrm{T}$ tumor). Graph of mean $\mathrm{pH}$ values for normal tissue and tumor (d). Adapted with permission from Journal of the American Chemical Society 2018, 140, 5925-5933, doi:https://doi.org/ 10.1021/jacs.8b00277. Copyright 2018 American Chemical Society

\section{a}<smiles>[R7]N1CCN(c2ccc3c(c2)P(=O)(c2ccccc2)C2=CC(=[N+](CC)CC)C=CC2=C3c2c(C)cccc2C)CC1</smiles>

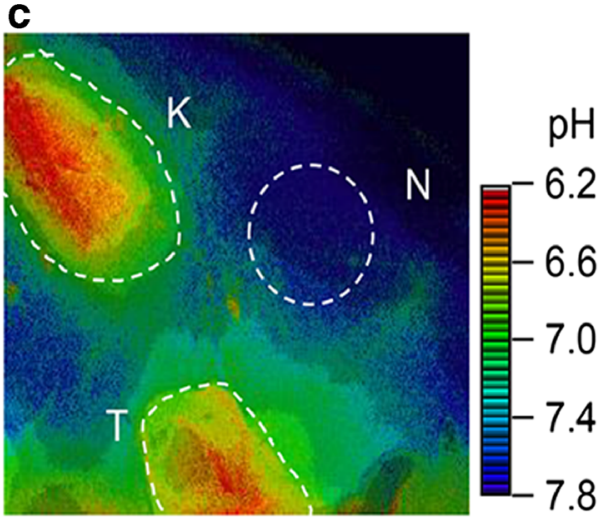

b
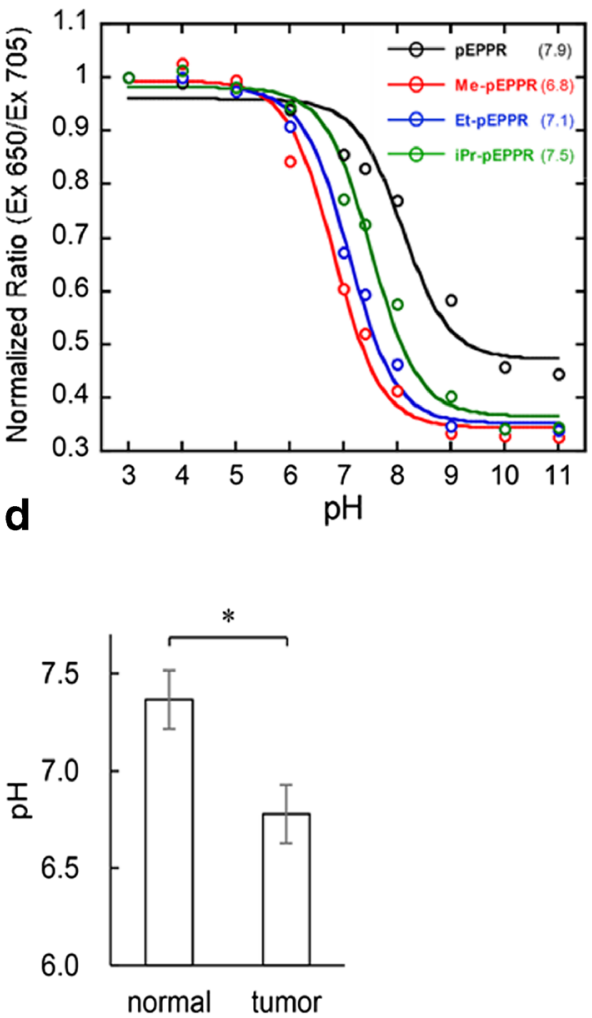
In the acidic conditions of the tumor microenvironment, the cleavage of $\mathrm{pH}$ liable linkage with the concomitant disruption of aggregates results in a remarkable fluorescence enhancement with a high tumor/background ratio. The ratio changed more dramatically upon time in a more metastatic hepatic tumor model (HCCLM3-GFP) in comparison to a less metastatic one (HepG2) [142].

\section{Photoacoustic imaging}

Optoacoustic or photoacoustic imaging (PAI) combines optical absorption with ultrasound detection for providing high spatial and temporal resolution images [143, 144]. Since highly penetrant soundwaves are detected instead of light, optoacoustic imaging has greater penetration depth than optical imaging modalities [145]. Moreover, optoacoustic imaging offers greater specificity than conventional ultrasound due to detection of ultrasound emissions from light-absorbing chromophore-containing molecules, which can be exogenous probes, as well as endogenous systems such as hemoglobin, melanin, and lipids [146-149]. The ideal optoacoustic contrast agent should be characterized by a strong absorption in the optical window to maximize penetration depth and by a low quantum yield (the ratio of the number of photons emitted to the number of photons absorbed), as the non-radiative conversion of light energy to heat is maximized [150]. The capability of providing $\mathrm{pH}$ information is dependent on the absorption/emission properties of the fluorescent dyes; therefore, ratiometric approaches for measuring tumor $\mathrm{pH}$ such as those described above have been developed also for PAI probes. This task has been accomplished by assembling a $\mathrm{pH}$-sensitive dye with a $\mathrm{pH}$-inert dye in a nanoprobe. Benzo- $\alpha$-phenoxazine (a pH-responsive dye) and IR825 (a pH-inert dye) can self-assemble onto human serum albumin (HSA) to form nanoparticles that allow assessment of tumor $\mathrm{pH}$ by ratioing signal intensities produced by excitations at 680 and $825 \mathrm{~nm}$, for $\mathrm{pH}$-sensitive and $\mathrm{pH}$-insensitive emissions, respectively [151]. In a similar way, the $\mathrm{pH}$-responsive near-infrared croconine dye can induce self-assembly on serum albumin nanoparticles for real-time ratiometric (680 and $810 \mathrm{~nm})$ optoacoustic tumor $\mathrm{pH}$ imaging [152]. Other classes of nanosystems can be exploited by this approach, such as semiconducting oligomers that, following the binding with the $\mathrm{pH}$-responsive boron-dipyrromethene dye, which can provide tumor $\mathrm{pH}$ mapping by ratioing the measured optoacoustic signals at 680 and $750 \mathrm{~nm}$. It has been shown that this method readily distinguishes healthy and tumor tissues [153]. Polyacrylamide nanoparticles encapsulated with an optical $\mathrm{pH}$ indicator (SNARF-5F) have
Fig. 15 Photoacoustic imaging of tumor $\mathrm{pH}$. Spectral properties of SNARF-5F encapsulated polyacrylamide based nanoparticle (NP) at different $\mathrm{pH}$ values (a). Boxplot showing the measured $\mathrm{pH}$ levels in tumor and healthy tissues (b) and quantitative PAI $\mathrm{pH}$ maps after SNARF-PAA NP injection in healthy (c) and in tumor (d) tissues overlaid to B-mode ultrasound images. Adapted with permission from Nature Communications 2017, 8, 471
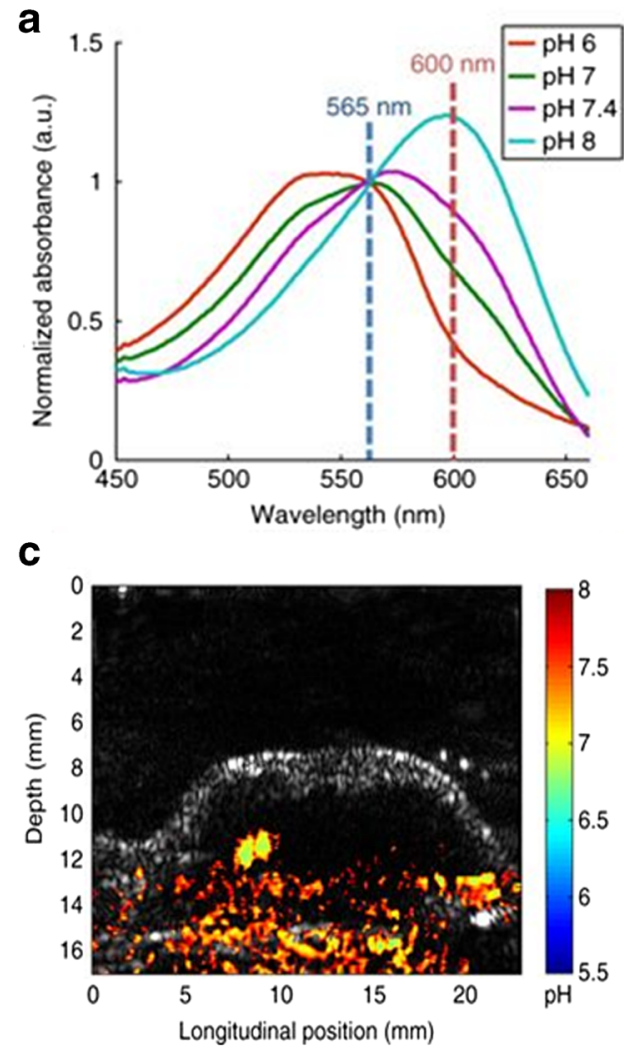

b

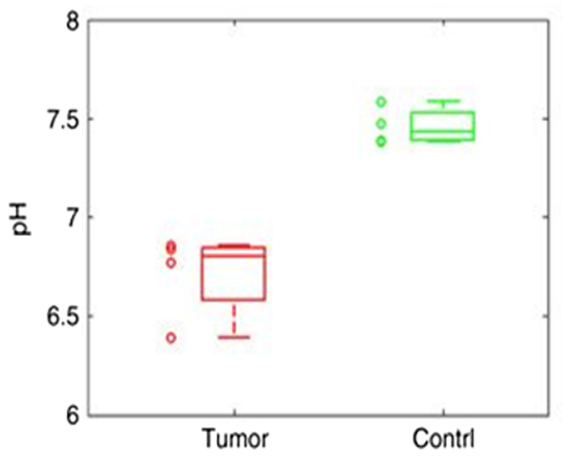

d

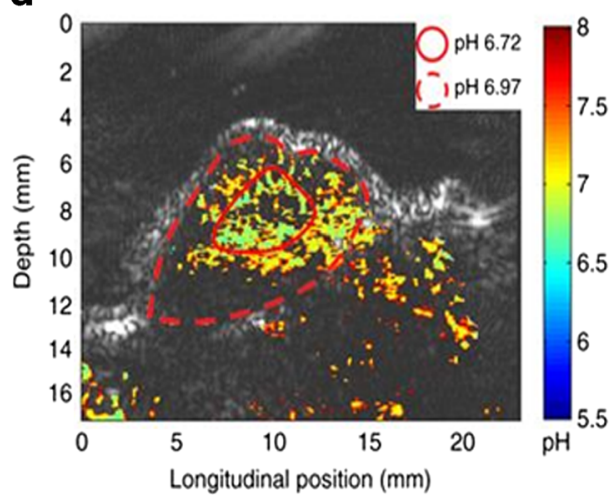


also been reported as $\mathrm{pH}$-sensing nanoprobes. The capability of fast multi-wavelength PAI combined with spectral unmixing techniques can provide accurate tumor $\mathrm{pH}$ measurements not susceptible to the background optical absorption of endogenous biomolecules, i.e., oxy- and deoxy-hemoglobin (Fig. 15) [154].

The major limitations of these probes are related to the stability of the self-assembled nanoparticles that usually require further conjugation via glutaraldehyde to induce covalent cross-linking and to the toxicity profiles and biodistribution properties that need to be further optimized for their clinical translation. In addition, the nonlinear response of the ratiometric curve to changes in $\mathrm{pH}$, coupled to the nonlinear optical properties of the photoacoustic effect, makes them more useful for detecting rapid or relative $\mathrm{pH}$ changes, rather than $\mathrm{pH}$ values. Despite these limitations, optoacoustic $\mathrm{pH}$ imaging overcomes the penetration depth limit of optical imaging, so it has the potential to measure tumor $\mathrm{pH}$ in vivo in superficial tumors up to 5-7-mm depth or in endoscopically accessible tumors at clinical level.

\section{Summary}

A wide range of imaging-based techniques have been investigated to monitor tumor $\mathrm{pH}$. In the last decade, several approaches have solved the limitations associated with first studies, including spatial and temporal resolution, $\mathrm{pH}$ sensitivity, and clinical translatability. To date, among the proposed techniques, MRI and in particular MRI-CEST methods have emerged as those endowed with good $\mathrm{pH}$ sensitivity for assessing tumor acidosis and $\mathrm{pH}$ changes following therapeutic treatments and with high spatial resolution for evaluating heterogeneity of the extracellular acidification. In addition, MRI-CEST using Iopamidol has been used to map tumor $\mathrm{pH}$ at clinical level, thus providing a novel imaging protocol for assessing fundamental questions in tumor biology, for evaluating the relationship between tumor acidosis and aggressiveness, and for monitoring treatment response to novel anticancer therapies. However, critical evaluation of this approach in single-site and multi-site studies should be encouraged to validate the clinical utility of imaging tumor acidosis as a novel noninvasive diagnostic tool.

Acknowledgements We thank Professor Silvio Aime for his helpful suggestions.

Funding information This work was supported by grants from the Associazione Italiana Ricerca Cancro (AIRC MFAG 2017 - ID. 20153 project - P.I. Longo Dario Livio), from Compagnia San Paolo project (Regione Piemonte, Grant No. CSTO165925), and from the European Union's Horizon 2020 research and innovation programme (Grant Agreement No. 667510). Lorena Consolino was supported by the Fondazione Umberto Veronesi fellowship program.

\section{Compliance with ethical standards}

Conflict of interest The authors declare that they have no conflict of interest.

Open Access This article is distributed under the terms of the Creative Commons Attribution 4.0 International License (http:// creativecommons.org/licenses/by/4.0/), which permits unrestricted use, distribution, and reproduction in any medium, provided you give appropriate credit to the original author(s) and the source, provide a link to the Creative Commons license, and indicate if changes were made.

Publisher's note Springer Nature remains neutral with regard to jurisdictional claims in published maps and institutional affiliations.

\section{References}

1. Denko, N. C. (2008). Hypoxia, HIF1 and glucose metabolism in the solid tumour. Nature Reviews. Cancer, 8(9), 705-713. https:// doi.org/10.1038/nrc2468.

2. Shaw, R. J. (2006). Glucose metabolism and cancer. Current Opinion in Cell Biology, 18(6), 598-608. https://doi.org/10.1016/j.ceb.2006. 10.005 .

3. Warburg, O. (1956). On the origin of cancer cells. Science, 123(3191), 309-314.

4. Gatenby, R. A., \& Gillies, R. J. (2004). Why do cancers have high aerobic glycolysis? Nature Reviews. Cancer, 4(11), 891-899. https://doi.org/10.1038/nrc1478.

5. Schornack, P. A., \& Gillies, R. J. (2003). Contributions of cell metabolism and $\mathrm{H}+$ diffusion to the acidic $\mathrm{pH}$ of tumors. Neoplasia, 5(2), 135-145.

6. Granja, S., Tavares-Valente, D., Queiros, O., \& Baltazar, F. (2017). Value of $\mathrm{pH}$ regulators in the diagnosis, prognosis and treatment of cancer. Seminars in Cancer Biology, 43, 17-34. https://doi.org/10.1016/j.semcancer.2016.12.003.

7. Kato, Y., Ozawa, S., Miyamoto, C., Maehata, Y., Suzuki, A., Maeda, T., \& Baba, Y. (2013). Acidic extracellular microenvironment and cancer. Cancer Cell International, 13(1), 89. https://doi. org/10.1186/1475-2867-13-89.

8. Estrella, V., Chen, T., Lloyd, M., Wojtkowiak, J., Cornnell, H. H., Ibrahim-Hashim, A., Bailey, K., Balagurunathan, Y., Rothberg, J. M., Sloane, B. F., Johnson, J., Gatenby, R. A., \& Gillies, R. J. (2013). Acidity generated by the tumor microenvironment drives local invasion. Cancer Research, 73(5), 1524-1535. https://doi. org/10.1158/0008-5472.CAN-12-2796.

9. Gatenby, R. A., Gawlinski, E. T., Gmitro, A. F., Kaylor, B., \& Gillies, R. J. (2006). Acid-mediated tumor invasion: a multidisciplinary study. Cancer Research, 66(10), 5216-5223. https://doi. org/10.1158/0008-5472.CAN-05-4193.

10. Kolosenko, I., Avnet, S., Baldini, N., Viklund, J., \& De Milito, A. (2017). Therapeutic implications of tumor interstitial acidification. Seminars in Cancer Biology, 43, 119-133. https://doi.org/10. 1016/j.semcancer.2017.01.008.

11. Neri, D., \& Supuran, C. T. (2011). Interfering with $\mathrm{pH}$ regulation in tumours as a therapeutic strategy. Nature Reviews. Drug Discovery, 10(10), 767-777. https://doi.org/10.1038/nrd3554.

12. Harguindey, S., Arranz, J. L., Wahl, M. L., Orive, G., \& Reshkin, S. J. (2009). Proton transport inhibitors as potentially selective anticancer drugs. Anticancer Research, 29(6), 2127-2136.

13. Rata, M., Giles, S. L., deSouza, N. M., Leach, M. O., \& Payne, G. S. (2014). Comparison of three reference methods for the measurement of intracellular $\mathrm{pH}$ using 31P MRS in healthy volunteers 
and patients with lymphoma. NMR in Biomedicine, 27(2), 158162.

14. Stubbs, M., Bhujwalla, Z. M., Tozer, G. M., Rodrigues, L. M., Maxwell, R. J., Morgan, R., Howe, F. A., \& Griffiths, J. R. (1992). An assessment of 31P MRS as a method of measuring $\mathrm{pH}$ in rat tumours. NMR in Biomedicine, 5(6), 351-359.

15. Soto, G. E., Zhu, Z., Evelhoch, J. L., \& Ackerman, J. J. (1996). Tumor 31P NMR pH measurements in vivo: a comparison of inorganic phosphate and intracellular 2-deoxyglucose-6phosphate as $\mathrm{pHnmr}$ indicators in murine radiation-induced fibrosarcoma-1. Magnetic Resonance in Medicine, 36(5), 698-704.

16. Madden, A., Glaholm, J., \& Leach, M. O. (1990). An assessment of the sensitivity of in vivo 31P nuclear magnetic resonance spectroscopy as a means of detecting $\mathrm{pH}$ heterogeneity in tumours: a simulation study. British Journal of Radiology, 63(746), 120-124. https://doi.org/10.1259/0007-1285-63-746-120.

17. Gillies, R. J., Liu, Z., \& Bhujwalla, Z. (1994). 31P-MRS measurements of extracellular pH of tumors using 3aminopropylphosphonate. The American Journal of Physiology, 267(1 Pt 1), C195-C203. https://doi.org/10.1152/ajpcell.1994. 267.1.C195.

18. Lutz, N. W., Le Fur, Y., Chiche, J., Pouyssegur, J., \& Cozzone, P. J. (2013). Quantitative in vivo characterization of intracellular and extracellular $\mathrm{pH}$ profiles in heterogeneous tumors: a novel method enabling multiparametric $\mathrm{pH}$ analysis. Cancer Research, 73(15), 4616-4628. https://doi.org/10.1158/0008-5472.CAN-13-0767.

19. Gerweck, L. E., \& Seetharaman, K. (1996). Cellular pH gradient in tumor versus normal tissue: potential exploitation for the treatment of cancer. Cancer Research, 56(6), 1194-1198.

20. Raghunand, N., Mahoney, B., van Sluis, R., Baggett, B., \& Gillies, R. J. (2001). Acute metabolic alkalosis enhances response of $\mathrm{C} 3 \mathrm{H}$ mouse mammary tumors to the weak base mitoxantrone. Neoplasia, 3(3), 227-235. https://doi.org/10.1038/sj/neo/ 7900151.

21. Raghunand, N., He, X., van Sluis, R., Mahoney, B., Baggett, B., Taylor, C. W., et al. (1999). Enhancement of chemotherapy by manipulation of tumour pH. British Journal of Cancer, 80(7), 1005-1011. https://doi.org/10.1038/sj.bjc.6690455.

22. Zhou, R., Bansal, N., Leeper, D. B., Pickup, S., \& Glickson, J. D. (2001). Enhancement of hyperglycemia-induced acidification of human melanoma xenografts with inhibitors of respiration and ion transport. Academic Radiology, 8(7), 571-582. https://doi.org/10. 1016/S1076-6332(03)80681-5.

23. Wenger, K. J., Hattingen, E., Franz, K., Steinbach, J. P., Bahr, O., \& Pilatus, U. (2017). Intracellular pH measured by (31) P-MRspectroscopy might predict site of progression in recurrent glioblastoma under antiangiogenic therapy. Journal of Magnetic Resonance Imaging, 46(4), 1200-1208. https://doi.org/10.1002/ jmri.25619.

24. Yu, J. X., Hallac, R. R., Chiguru, S., \& Mason, R. P. (2013). New frontiers and developing applications in 19F NMR. Progress in Nuclear Magnetic Resonance Spectroscopy, 70, 25-49. https:// doi.org/10.1016/j.pnmrs.2012.10.001.

25. Mason, R. P. (1999). Transmembrane $\mathrm{pH}$ gradients in vivo: measurements using fluorinated vitamin B6 derivatives. Current Medicinal Chemistry, 6(6), 481-499.

26. Yu, J. X., Cui, W., Bourke, V. A., \& Mason, R. P. (2012). 6Trifluoromethylpyridoxine: novel (19)F NMR $\mathrm{pH}$ indicator for in vivo detection. Journal of Medicinal Chemistry, 55(15), 6814-6821. https://doi.org/10.1021/jm300520q.

27. Frenzel, T., Kossler, S., Bauer, H., Niedballa, U., \& Weinmann, H. J. (1994). Noninvasive in vivo $\mathrm{pH}$ measurements using a fluorinated $\mathrm{pH}$ probe and fluorine-19 magnetic resonance spectroscopy. Investigative Radiology, 29(Suppl 2), S220-S222.

28. Oishi, M., Sumitani, S., \& Nagasaki, Y. (2007). On-off regulation of $19 \mathrm{~F}$ magnetic resonance signals based on $\mathrm{pH}$-sensitive
PEGylated nanogels for potential tumor-specific smart 19F MRI probes. Bioconjugate Chemistry, 18(5), 1379-1382. https://doi. org/10.1021/bc7002154.

29. Huang, X., Huang, G., Zhang, S., Sagiyama, K., Togao, O., Ma, X., Wang, Y., Li, Y., Soesbe, T. C., Sumer, B. D., Takahashi, M. Sherry, A. D., \& Gao, J. (2013). Multi-chromatic pH-activatable 19F-MRI nanoprobes with binary ON/OFF $\mathrm{pH}$ transitions and chemical-shift barcodes. Angewandte Chemie (International Ed. in English), 52(31), 8074-8078. https://doi.org/10.1002/anie. 201301135.

30. Aime, S., Botta, M., Milone, L., \& Terreno, E. (1996). Paramagnetic complexes as novel NMR pH indicators. Chemical Communications, (11), 1265-1266. https://doi.org/10. 1039/cc9960001265.

31. Vermathen, P., Capizzano, A. A., \& Maudsley, A. A. (2000). Administration and (1)H MRS detection of histidine in human brain: application to in vivo $\mathrm{pH}$ measurement. Magnetic Resonance in Medicine, 43(5), 665-675.

32. van Sluis, R., Bhujwalla, Z. M., Raghunand, N., Ballesteros, P., Alvarez, J., Cerdan, S., et al. (1999). In vivo imaging of extracellular pH using 1H MRSI. Magnetic Resonance in Medicine, 41(4), 743-750.

33. Garcia-Martin, M. L., Herigault, G., Remy, C., Farion, R., Ballesteros, P., Coles, J. A., et al. (2001). Mapping extracellular $\mathrm{pH}$ in rat brain gliomas in vivo by $1 \mathrm{H}$ magnetic resonance spectroscopic imaging: comparison with maps of metabolites. Cancer Research, 61(17), 6524-6531.

34. Provent, P., Benito, M., Hiba, B., Farion, R., Lopez-Larrubia, P., Ballesteros, P., Remy, C., Segebarth, C., Cerdan, S., Coles, J. A., \& Garcia-Martin, M. L. (2007). Serial in vivo spectroscopic nuclear magnetic resonance imaging of lactate and extracellular $\mathrm{pH}$ in rat gliomas shows redistribution of protons away from sites of glycolysis. Cancer Research, 67(16), 7638-7645. https://doi.org/ 10.1158/0008-5472.CAN-06-3459.

35. Lee, S. H., Mcintyre, D., Honess, D., Hulikova, A., PachecoTorres, J., Cerdan, S., et al. (2018). Carbonic anhydrase IX is a $\mathrm{pH}$-stat that sets an acidic tumour extracellular $\mathrm{pH}$ in vivo. British Journal of Cancer, 119(5), 622-630. https://doi.org/10.1038/ s41416-018-0216-5.

36. Hashim, A. I., Cornnell, H. H., Ribeiro, M. D. C., Abrahams, D., Cunningham, J., Lloyd, M., et al. (2011). Reduction of metastasis using a non-volatile buffer. Clinical and Experimental Metastasis, 28(8), 841-849. https://doi.org/10.1007/s10585-011-9415-7.

37. Soler-Padros, J., Perez-Mayoral, E., Dominguez, L., LopezLarrubia, P., Soriano, E., Marco-Contelles, J. L., et al. (2007). Novel generation of $\mathrm{pH}$ indicators for proton magnetic resonance spectroscopic imaging. Journal of Medicinal Chemistry, 50(18), 4539-4542. https://doi.org/10.1021/jm070044j.

38. Sherry, A. D., Caravan, P., \& Lenkinski, R. E. (2009). Primer on gadolinium chemistry. Journal of Magnetic Resonance Imaging, 30(6), 1240-1248. https://doi.org/10.1002/jmri.21966.

39. Aime, S., Botta, M., \& Terreno, E. (2005). Gd(III)-based contrast agents for MRI. Advances in Inorganic Chemistry - Including Bioinorganic Studies, 57(57), 173-237. https://doi.org/10.1016/ S0898-8838(05)57004-1.

40. Botta, M., \& Tei, L. (2012). Relaxivity enhancement in macromolecular and nanosized GdIII-based MRI contrast agents. European Journal of Inorganic Chemistry, 2012(12), 1945-1960. https:// doi.org/10.1002/ejic.201101305.

41. Burtea, C., Laurent, S., Vander Elst, L., \& Muller, R. N. (2008). Contrast agents: magnetic resonance. Handb Exp Pharmacol(185 Pt 1), 135-165, doi:https://doi.org/10.1007/978-3-540-72718-77.

42. Caravan, P., Ellison, J. J., McMurry, T. J., \& Lauffer, R. B. (1999). Gadolinium(III) chelates as MRI contrast agents: Structure, dynamics, and applications. Chemical Reviews, 99(9), 2293-2352. 
43. Aime, S., Frullano, L., \& Geninatti Crich, S. (2002). Compartmentalization of a gadolinium complex in the apoferritin cavity: a route to obtain high relaxivity contrast agents for magnetic resonance imaging. Angewandte Chemie (International Ed. in English), 41(6), 1017-1019.

44. Zhang, S., Wu, K., \& Sherry, A. D. (1999). A novel pH-sensitive MRI contrast agent. Angewandte Chemie (International Ed. in English), 38(21), 3192-3194.

45. Raghunand, N., Howison, C., Sherry, A. D., Zhang, S., \& Gillies, R. J. (2003). Renal and systemic $\mathrm{pH}$ imaging by contrastenhanced MRI. Magnetic Resonance in Medicine, 49(2), 249257. https://doi.org/10.1002/mrm.10347.

46. Garcia-Martin, M. L., Martinez, G. V., Raghunand, N., Sherry, A. D., Zhang, S., \& Gillies, R. J. (2006). High resolution pH(e) imaging of rat glioma using $\mathrm{pH}$-dependent relaxivity. Magnetic Resonance in Medicine, 55(2), 309-315. https://doi.org/10.1002/ mrm.20773.

47. Martinez, G. V., Zhang, X., Garcia-Martin, M. L., Morse, D. L., Woods, M., Sherry, A. D., et al. (2011). Imaging the extracellular $\mathrm{pH}$ of tumors by MRI after injection of a single cocktail of T1 and T2 contrast agents. NMR in Biomedicine, 24(10), 1380-1391. https://doi.org/10.1002/nbm.1701.

48. Aime, S., Fedeli, F., Sanino, A., \& Terreno, E. (2006). A R2/R1 ratiometric procedure for a concentration-independent, $\mathrm{pH}-\mathrm{re}$ sponsive, Gd(III)-based MRI agent. Journal of the American Chemical Society, 128(35), 11326-11327. https://doi.org/10. 1021/ja062387x.

49. Gianolio, E., Porto, S., Napolitano, R., Baroni, S., Giovenzana, G. B., \& Aime, S. (2012). Relaxometric investigations and MRI evaluation of a liposome-loaded $\mathrm{pH}$-responsive gadolinium(III) complex. Inorganic Chemistry, 51(13), 7210-7217. https://doi.org/10. $1021 /$ ic 300447n.

50. Gianolio, E., Napolitano, R., Fedeli, F., Arena, F., \& Aime, S. (2009). Poly-beta-cyclodextrin based platform for $\mathrm{pH}$ mapping via a ratiometric F-19/H-1 MRI method. Chemical Communications, (40), 6044-6046. https://doi.org/10.1039/ b914540k.

51. Ward, K. M., Aletras, A. H., \& Balaban, R. S. (2000). A new class of contrast agents for MRI based on proton chemical exchange dependent saturation transfer (CEST). Journal of Magnetic Resonance, 143(1), 79-87. https://doi.org/10.1006/jmre.1999. 1956.

52. Vinogradov, E., Sherry, A. D., \& Lenkinski, R. E. (2013). CEST: from basic principles to applications, challenges and opportunities. Journal of Magnetic Resonance, 229, 155-172. https://doi.org/10. 1016/j.jmr.2012.11.024.

53. Liu, G., Song, X., Chan, K. W., \& McMahon, M. T. (2013). Nuts and bolts of chemical exchange saturation transfer MRI. NMR in Biomedicine, 26(7), 810-828. https://doi.org/10.1002/nbm.2899.

54. Zaiss, M., \& Bachert, P. (2013). Chemical exchange saturation transfer (CEST) and MR Z-spectroscopy in vivo: a review of theoretical approaches and methods. Physics in Medicine and Biology, 58(22), R221-R269. https://doi.org/10.1088/0031-9155/ 58/22/R221.

55. Ward, K. M., \& Balaban, R. S. (2000). Determination of pH using water protons and chemical exchange dependent saturation transfer (CEST). Magnetic Resonance in Medicine, 44(5), 799-802.

56. Hancu, I., Dixon, W. T., Woods, M., Vinogradov, E., Sherry, A. D., \& Lenkinski, R. E. (2010). CEST and PARACEST MR contrast agents. Acta Radiologica, 51(8), 910-923. https://doi.org/10. 3109/02841851.2010.502126.

57. Dastru, W., Longo, D., \& Aime, S. (2011). Contrast agents and mechanisms. Drug Discovery Today: Technologies, 8(2-4), e109e115. https://doi.org/10.1016/j.ddtec.2011.11.013.

58. Zhou, J., Payen, J. F., Wilson, D. A., Traystman, R. J., \& van Zijl, P. C. (2003). Using the amide proton signals of intracellular proteins and peptides to detect $\mathrm{pH}$ effects in MRI. Nature Medicine, 9(8), 1085-1090. https://doi.org/10.1038/nm907.

59. Sun, P. Z., Cheung, J. S., Wang, E., \& Lo, E. H. (2011). Association between $\mathrm{pH}$-weighted endogenous amide proton chemical exchange saturation transfer MRI and tissue lactic acidosis during acute ischemic stroke. Journal of Cerebral Blood Flow and Metabolism, 31(8), 1743-1750. https://doi.org/10. 1038/jcbfm.2011.23.

60. Harston, G. W., Tee, Y. K., Blockley, N., Okell, T. W., Thandeswaran, S., Shaya, G., et al. (2015). Identifying the ischaemic penumbra using $\mathrm{pH}$-weighted magnetic resonance imaging. Brain, 138(Pt 1), 36-42. https://doi.org/10.1093/brain/ awu374.

61. Sun, P. Z., Zhou, J., Sun, W., Huang, J., \& van Zijl, P. C. (2007). Detection of the ischemic penumbra using $\mathrm{pH}$-weighted MRI. Journal of Cerebral Blood Flow and Metabolism, 27(6), 11291136. https://doi.org/10.1038/sj.jcbfm.9600424.

62. Sun, P. Z., Wang, E., Cheung, J. S., Zhang, X., Benner, T., \& Sorensen, A. G. (2011). Simulation and optimization of pulsed radio frequency irradiation scheme for chemical exchange saturation transfer (CEST) MRI-demonstration of $\mathrm{pH}$-weighted pulsedamide proton CEST MRI in an animal model of acute cerebral ischemia. Magnetic Resonance in Medicine, 66(4), 1042-1048. https://doi.org/10.1002/mrm.22894.

63. Sun, P. Z., Benner, T., Copen, W. A., \& Sorensen, A. G. (2010), Early experience of translating $\mathrm{pH}$-weighted MRI to image human subjects at 3 Tesla. Stroke, 41(10 Suppl), S147-S151. https://doi. org/10.1161/STROKEAHA.110.595777.

64. Sun, P. Z., \& Sorensen, A. G. (2008). Imaging pH using the chemical exchange saturation transfer (CEST) MRI: correction of concomitant RF irradiation effects to quantify CEST MRI for chemical exchange rate and $\mathrm{pH}$. Magnetic Resonance in Medicine, 60(2), 390-397. https://doi.org/10.1002/mrm.21653.

65. Wu, Y., Zhou, I. Y., Lu, D., Manderville, E., Lo, E. H., Zheng, H., \& Sun, P. Z. (2018). pH-sensitive amide proton transfer effect dominates the magnetization transfer asymmetry contrast during acute ischemia-quantification of multipool contribution to in vivo CEST MRI. Magnetic Resonance in Medicine, 79(3), 1602-1608. https://doi.org/10.1002/mrm.26829.

66. McVicar, N., Li, A. X., Goncalves, D. F., Bellyou, M., Meakin, S. O., Prado, M. A., et al. (2014). Quantitative tissue pH measurement during cerebral ischemia using amine and amide concentration-independent detection (AACID) with MRI. Journal of Cerebral Blood Flow and Metabolism, 34(4), 690698. https://doi.org/10.1038/jcbfm.2014.12.

67. McVicar, N., Li, A. X., Meakin, S. O., \& Bartha, R. (2015). Imaging chemical exchange saturation transfer (CEST) effects following tumor-selective acidification using lonidamine. NMR in Biomedicine, 28(5), 566-575. https://doi.org/10.1002/nbm. 3287.

68. Marathe, K., McVicar, N., Li, A., Bellyou, M., Meakin, S., \& Bartha, R. (2016). Topiramate induces acute intracellular acidification in glioblastoma. Journal of Neuro-Oncology, 130(3), 465472. https://doi.org/10.1007/s11060-016-2258-y.

69. Albatany, M., Li, A., Meakin, S., \& Bartha, R. (2018). Dichloroacetate induced intracellular acidification in glioblastoma: in vivo detection using AACID-CEST MRI at 9.4 tesla. Journal of Neuro-Oncology, 136(2), 255-262. https://doi.org/10. 1007/s11060-017-2664-9.

70. Albatany, M., Meakin, S., \& Bartha, R. (2018). The Monocarboxylate transporter inhibitor quercetin induces intracellular acidification in a mouse model of glioblastoma multiforme: in-vivo detection using magnetic resonance imaging. Investigational New Drugs. https://doi.org/10.1007/s10637-0180644-3. 
71. Aime, S., Nano, R., \& Grandi, M. (1988). A new class of contrast agents for magnetic resonance imaging based on selective reduction of water-T2 by chemical exchange. Investigative Radiology, 23(Suppl 1), S267-S270.

72. Anemone, A., Consolino, L., \& Longo, D. L. (2017). MRI-CEST assessment of tumour perfusion using X-ray iodinated agents: comparison with a conventional Gd-based agent. European Radiology, 27(5), 2170-2179. https://doi.org/10.1007/s00330016-4552-7.

73. Longo, D. L., Michelotti, F., Consolino, L., Bardini, P., Digilio, G., Xiao, G., Sun, P. Z., \& Aime, S. (2016). In vitro and in vivo assessment of nonionic iodinated radiographic molecules as chemical exchange saturation transfer magnetic resonance imaging tumor perfusion agents. Investigative Radiology, 51(3), 155-162. https://doi.org/10.1097/RLI.0000000000000217.

74. Aime, S., Calabi, L., Biondi, L., De Miranda, M., Ghelli, S., Paleari, L., et al. (2005). Iopamidol: exploring the potential use of a well-established x-ray contrast agent for MRI. Magnetic Resonance in Medicine, 53(4), 830-834. https://doi.org/10.1002/ mrm.20441.

75. Longo, D. L., Dastru, W., Digilio, G., Keupp, J., Langereis, S., Lanzardo, S., et al. (2011). Iopamidol as a responsive MRIchemical exchange saturation transfer contrast agent for $\mathrm{pH}$ mapping of kidneys: In vivo studies in mice at 7 T. Magnetic Resonance in Medicine, 65(1), 202-211. https://doi.org/10.1002/ mrm.22608.

76. Sun, P. Z., Longo, D. L., Hu, W., Xiao, G., \& Wu, R. (2014). Quantification of iopamidol multi-site chemical exchange properties for ratiometric chemical exchange saturation transfer (CEST) imaging of $\mathrm{pH}$. Physics in Medicine and Biology, 59(16), 44934504. https://doi.org/10.1088/0031-9155/59/16/4493.

77. Longo, D. L., Busato, A., Lanzardo, S., Antico, F., \& Aime, S. (2013). Imaging the $\mathrm{pH}$ evolution of an acute kidney injury model by means of iopamidol, a MRI-CEST pH-responsive contrast agent. Magnetic Resonance in Medicine, 70(3), 859-864. https:// doi.org/10.1002/mrm.24513.

78. Longo, D. L., Cutrin, J. C., Michelotti, F., Irrera, P., \& Aime, S. (2017). Noninvasive evaluation of renal $\mathrm{pH}$ homeostasis after ischemia reperfusion injury by CEST-MRI. NMR in Biomedicine, 30(7). https://doi.org/10.1002/nbm.3720.

79. Longo, D. L., Bartoli, A., Consolino, L., Bardini, P., Arena, F., Schwaiger, M., \& Aime, S. (2016). In vivo imaging of tumor metabolism and acidosis by combining PET and MRI-CEST $\mathrm{pH}$ imaging. Cancer Research, 76(22), 6463-6470. https://doi.org/10. 1158/0008-5472.CAN-16-0825.

80. Peppicelli, S., Bianchini, F., \& Calorini, L. (2014). Extracellular acidity, a "reappreciated" trait of tumor environment driving malignancy: perspectives in diagnosis and therapy. Cancer and Metastasis Reviews, 33(2-3), 823-832. https://doi.org/10.1007/ s10555-014-9506-4.

81. Papandreou, I., Goliasova, T., \& Denko, N. C. (2011). Anticancer drugs that target metabolism: Is dichloroacetate the new paradigm? International Journal of Cancer, 128(5), 1001-1008. https://doi.org/10.1002/ijc.25728.

82. Anemone, A., Consolino, L., Conti, L., Reineri, F., Cavallo, F., Aime, S., \& Longo, D. L. (2017). In vivo evaluation of tumour acidosis for assessing the early metabolic response and onset of resistance to dichloroacetate by using magnetic resonance $\mathrm{pH}$ imaging. International Journal of Oncology, 51(2), 498-506. https:// doi.org/10.3892/ijo.2017.4029.

83. Chen, L. Q., Howison, C. M., Jeffery, J. J., Robey, I. F., Kuo, P. H., \& Pagel, M. D. (2014). Evaluations of extracellular $\mathrm{pH}$ within in vivo tumors using acidoCEST MRI. Magnetic Resonance in Medicine, 72(5), 1408-1417. https://doi.org/10.1002/mrm.25053.

84. Chen, L. Q., Randtke, E. A., Jones, K. M., Moon, B. F., Howison, C. M., \& Pagel, M. D. (2015). Evaluations of tumor acidosis within in vivo tumor models using parametric maps generated with Acido CEST MRI. Molecular Imaging and Biology, 17(4), 488-496. https://doi.org/10.1007/s11307-014-0816-2.

85. Moon, B. F., Jones, K. M., Chen, L. Q., Liu, P., Randtke, E. A., Howison, C. M., \& Pagel, M. D. (2015). A comparison of iopromide and iopamidol, two acidoCEST MRI contrast media that measure tumor extracellular pH. Contrast Media \& Molecular Imaging, 10(6), 446-455. https://doi.org/10.1002/ cmmi.1647.

86. Goldenberg, J. M., Cardenas-Rodriguez, J., \& Pagel, M. D. (2018). Preliminary results that assess metformin treatment in a preclinical model of pancreatic cancer using simultaneous [(18)F]FDG PET and acidoCEST MRI. Molecular Imaging and Biology, 20(4), 575-583. https://doi.org/10.1007/s11307-0181164-4.

87. Muller-Lutz, A., Khalil, N., Schmitt, B., Jellus, V., Pentang, G., Oeltzschner, G., et al. (2014). Pilot study of Iopamidol-based quantitative $\mathrm{pH}$ imaging on a clinical 3T MR scanner. MAGMA, 27(6), 477-485. https://doi.org/10.1007/s10334-014-0433-8.

88. Jones, K. M., Randtke, E. A., Yoshimaru, E. S., Howison, C. M., Chalasani, P., Klein, R. R., Chambers, S. K., Kuo, P. H., \& Pagel, M. D. (2017). Clinical translation of tumor acidosis measurements with AcidoCEST MRI. Molecular Imaging and Biology, 19(4), 617-625. https://doi.org/10.1007/s11307-016-1029-7.

89. Longo, D. L., Sun, P. Z., Consolino, L., Michelotti, F. C., Uggeri, F., \& Aime, S. (2014). A general MRI-CEST ratiometric approach for $\mathrm{pH}$ imaging: demonstration of in vivo $\mathrm{pH}$ mapping with iobitridol. Journal of the American Chemical Society, 136(41), 14333-14336. https://doi.org/10.1021/ja5059313.

90. Arena, F., Irrera, P., Consolino, L., Colombo Serra, S., Zaiss, M., \& Longo, D. L. (2018). Flip-angle based ratiometric approach for pulsed CEST-MRI pH imaging. Journal of Magnetic Resonance, 287, 1-9. https://doi.org/10.1016/j.jmr.2017.12.007.

91. Yang, X., Song, X., Ray Banerjee, S., Li, Y., Byun, Y., Liu, G., Bhujwalla, Z. M., Pomper, M. G., \& McMahon, M. T. (2016). Developing imidazoles as CEST MRI pH sensors. Contrast Media \& Molecular Imaging, 11(4), 304-312. https://doi.org/10. 1002/cmmi.1693.

92. Sherry, A. D., \& Wu, Y. (2013). The importance of water exchange rates in the design of responsive agents for MRI. Current Opinion in Chemical Biology, 17(2), 167-174. https://doi.org/10. 1016/j.cbpa.2012.12.012.

93. Aime, S., Barge, A., Delli Castelli, D., Fedeli, F., Mortillaro, A., Nielsen, F. U., \& Terreno, E. (2002). Paramagnetic lanthanide(III) complexes as $\mathrm{pH}$-sensitive chemical exchange saturation transfer (CEST) contrast agents for MRI applications. Magnetic Resonance in Medicine, 47(4), 639-648.

94. Aime, S., Delli Castelli, D., \& Terreno, E. (2002). Novel pHreporter MRI contrast agents. Angewandte Chemie. International Ed. In English, 41(22), 4334-4336.

95. Delli Castelli, D., Terreno, E., \& Aime, S. (2011). Yb(III)HPDO3A: a dual pH- and temperature-responsive CEST agent. Angewandte Chemie (International Ed. in English), 50(8), 1798 1800. https://doi.org/10.1002/anie.201007105.

96. Delli Castelli, D., Ferrauto, G., Cutrin, J. C., Terreno, E., \& Aime, S. (2014). In vivo maps of extracellular $\mathrm{pH}$ in murine melanoma by CEST-MRI. Magnetic Resonance in Medicine, 71(1), 326332. https://doi.org/10.1002/mrm.24664.

97. Ferrauto, G., Di Gregorio, E., Auboiroux, V., Petit, M., Berger, F., Aime, S., et al. (2018). CEST-MRI for glioma $\mathrm{pH}$ quantification in mouse model: validation by immunohistochemistry. NMR in Biomedicine, 31(11), e4005. https://doi.org/10.1002/nbm.4005.

98. Rancan, G., Delli Castelli, D., \& Aime, S. (2016). MRI CEST at 1T with large microeff $\mathrm{Ln}(3+)$ complexes $\mathrm{T} \mathrm{m}(3+)$-HPDO3A: an efficient MRI $\mathrm{pH}$ reporter. Magnetic Resonance in Medicine, 75(1), 329-336. https://doi.org/10.1002/mrm.25589. 
99. Liu, G., Li, Y., Sheth, V. R., \& Pagel, M. D. (2012). Imaging in vivo extracellular $\mathrm{pH}$ with a single paramagnetic chemical exchange saturation transfer magnetic resonance imaging contrast agent. Molecular Imaging, 11(1), 47-57.

100. Wang, X., Wu, Y., Soesbe, T. C., Yu, J., Zhao, P., Kiefer, G. E., \& Sherry, A. D. (2015). A pH-responsive MRI agent that can be activated beyond the tissue magnetization transfer window. Angewandte Chemie. International Ed. In English, 54(30), 8662-8664. https://doi.org/10.1002/anie.201502497.

101. Wu, Y., Zhang, S., Soesbe, T. C., Yu, J., Vinogradov, E., Lenkinski, R. E., \& Sherry, A. D. (2016). pH imaging of mouse kidneys in vivo using a frequency-dependent paraCEST agent. Magnetic Resonance in Medicine, 75(6), 2432-2441. https://doi. org/10.1002/mrm.25844.

102. Coman, D., Kiefer, G. E., Rothman, D. L., Sherry, A. D., \& Hyder, F. (2011). A lanthanide complex with dual biosensing properties: CEST (chemical exchange saturation transfer) and BIRDS (biosensor imaging of redundant deviation in shifts) with europium DOTA-tetraglycinate. NMR in Biomedicine, 24(10), 1216-1225. https://doi.org/10.1002/nbm.1677.

103. Huang, Y., Coman, D., Ali, M. M., \& Hyder, F. (2015). Lanthanide ion (III) complexes of $1,4,7,10$ tetraazacyclododecane-1,4,7,10-tetraaminophosphonate for dual biosensing of $\mathrm{pH}$ with chemical exchange saturation transfer (CEST) and biosensor imaging of redundant deviation in shifts (BIRDS). Contrast Media \& Molecular Imaging, 10(1), 51-58. https://doi.org/10.1002/cmmi.1604.

104. Coman, D., Huang, Y., Rao, J. U., De Feyter, H. M., Rothman, D. L., Juchem, C., et al. (2016). Imaging the intratumoral-peritumoral extracellular $\mathrm{pH}$ gradient of gliomas. NMR in Biomedicine, 29(3), 309-319. https://doi.org/10.1002/nbm.3466.

105. Rane, S., Spear, J. T., Zu, Z., Donahue, M. J., \& Gore, J. C. (2014). Functional MRI using spin lock editing preparation pulses. Magnetic Resonance Imaging, 32(7), 813-818. https://doi.org/ 10.1016/j.mri.2014.04.001.

106. Jin, T., Autio, J., Obata, T., \& Kim, S. G. (2011). Spin-locking versus chemical exchange saturation transfer MRI for investigating chemical exchange process between water and labile metabolite protons. Magnetic Resonance in Medicine, 65(5), 1448-1460. https://doi.org/10.1002/mrm.22721.

107. Jin, T., Iordanova, B., Hitchens, T. K., Modo, M., Wang, P., Mehrens, H., \& Kim, S. G. (2018). Chemical exchange-sensitive spin-lock (CESL) MRI of glucose and analogs in brain tumors. Magnetic Resonance in Medicine, 80(2), 488-495. https://doi.org/ 10.1002/mrm. 27183 .

108. Roeloffs, V., Meyer, C., Bachert, P., \& Zaiss, M. (2015). Towards quantification of pulsed spinlock and CEST at clinical MR scanners: an analytical interleaved saturation-relaxation (ISAR) approach. NMR in Biomedicine, 28(1), 40-53. https://doi.org/10. 1002/nbm.3192.

109. Cobb, J. G., Xie, J., Li, K., Gochberg, D. F., \& Gore, J. C. (2012). Exchange-mediated contrast agents for spin-lock imaging. Magnetic Resonance in Medicine, 67(5), 1427-1433. https://doi. org $/ 10.1002 / \mathrm{mrm} .23130$.

110. Zu, Z., Li, H., Jiang, X., \& Gore, J. C. (2018). Spin-lock imaging of exogenous exchange-based contrast agents to assess tissue $\mathrm{pH}$. Magnetic Resonance in Medicine, 79(1), 298-305. https://doi.org/ $10.1002 / \mathrm{mrm} .26681$.

111. Serrao, E. M., \& Brindle, K. M. (2016). Potential clinical roles for metabolic imaging with hyperpolarized [1-(13)C]pyruvate. Frontiers in Oncology, 6, 59. https://doi.org/10.3389/fonc.2016. 00059.

112. Nelson, S. J., Vigneron, D., Kurhanewicz, J., Chen, A., Bok, R., \& Hurd, R. (2008). DNP-hyperpolarized C magnetic resonance metabolic imaging for cancer applications. Applied Magnetic
Resonance, 34(3-4), 533-544. https://doi.org/10.1007/s00723008-0136-2.

113. Ardenkjaer-Larsen, J. H., Fridlund, B., Gram, A., Hansson, G., Hansson, L., Lerche, M. H., Servin, R., Thaning, M., \& Golman, K. (2003). Increase in signal-to-noise ratio of $>10,000$ times in liquid-state NMR. Proceedings of the National Academy of Sciences of the United States of America, 100(18), 1015810163. https://doi.org/10.1073/pnas.1733835100.

114. Viale, A., Reineri, F., Dastru, W., \& Aime, S. (2012). Hyperpolarized (13)C-pyruvate magnetic resonance imaging in cancer diagnostics. Expert Opin Med Diagn, 6(4), 335-345. https://doi.org/10.1517/17530059.2012.687372.

115. Gallagher, F. A., Kettunen, M. I., Day, S. E., Hu, D. E., Ardenkjaer-Larsen, J. H., Zandt, R., et al. (2008). Magnetic resonance imaging of $\mathrm{pH}$ in vivo using hyperpolarized 13C-labelled bicarbonate. Nature, 453(7197), 940-943. https://doi.org/10. 1038/nature07017.

116. Lim, H., Albatany, M., Martinez-Santiesteban, F., Bartha, R., \& Scholl, T. J. (2018). Longitudinal measurements of intra- and extracellular $\mathrm{pH}$ gradient in a rat model of glioma. Tomography, 4(2), 46-54. https://doi.org/10.18383/j.tom.2018.00001.

117. Duwel, S., Hundshammer, C., Gersch, M., Feuerecker, B., Steiger, K., Buck, A., et al. (2017). Imaging of $\mathrm{pH}$ in vivo using hyperpolarized (13)C-labelled zymonic acid. Nature Communications, 8, 15126. https://doi.org/10.1038/ ncomms 15126.

118. Hundshammer, C., Duwel, S., Kocher, S. S., Gersch, M., Feuerecker, B., Scheurer, C., et al. (2017). Deuteration of hyperpolarized (13) C-labeled zymonic acid enables sensitivityenhanced dynamic MRI of pH. Chemphyschem, 18(18), 24222425. https://doi.org/10.1002/cphc.201700779.

119. Cavallari, E., Carrera, C., Aime, S., \& Reineri, F. (2018). Metabolic studies of tumor cells using [1-(13) C] pyruvate hyperpolarized by means of PHIP-side arm hydrogenation. Chemphyschem, 20, 318-325. https://doi.org/10.1002/cphc. 201800652.

120. Reineri, F., Boi, T., \& Aime, S. (2015). ParaHydrogen induced polarization of $13 \mathrm{C}$ carboxylate resonance in acetate and pyruvate. Nature Communications, 6, 5858. https://doi.org/10.1038/ ncomms6858.

121. Danhier, P., \& Gallez, B. (2015). Electron paramagnetic resonance: a powerful tool to support magnetic resonance imaging research. Contrast Media \& Molecular Imaging, 10(4), 266281. https://doi.org/10.1002/cmmi.1630.

122. Hyodo, F., Matsumoto, S., Devasahayam, N., Dharmaraj, C., Subramanian, S., Mitchell, J. B., \& Krishna, M. C. (2009). Pulsed EPR imaging of nitroxides in mice. Journal of Magnetic Resonance, 197(2), 181-185. https://doi.org/10.1016/j.jmr.2008. 12.018 .

123. Lurie, D. J., Bussell, D. M., Bell, L. H., \& Mallard, J. R. (1988). Proton electron double magnetic-resonance imaging of freeradical solutions. Journal of Magnetic Resonance, 76(2), 366370. https://doi.org/10.1016/0022-2364(88)90123-0.

124. Kishimoto, S., Krishna, M. C., Khramtsov, V. V., Utsumi, H., \& Lurie, D. J. (2018). In vivo application of proton-electron doubleresonance imaging. Antioxidants \& Redox Signaling, 28(15), 1345-1364. https://doi.org/10.1089/ars.2017.7341.

125. Bobko, A. A., Eubank, T. D., Voorhees, J. L., Efimova, O. V., Kirilyuk, I. A., Petryakov, S., Trofimiov, D. G., Marsh, C. B., Zweier, J. L., Grigor'ev, I. A., Samouilov, A., \& Khramtsov, V. V. (2012). In vivo monitoring of $\mathrm{pH}$, redox status, and glutathione using L-band EPR for assessment of therapeutic effectiveness in solid tumors. Magnetic Resonance in Medicine, 67(6), 18271836. https://doi.org/10.1002/mrm.23196.

126. Bobko, A. A., Dhimitruka, I., Komarov, D. A., \& Khramtsov, V. V. (2012). Dual-function $\mathrm{pH}$ and oxygen phosphonated trityl 
probe. Analytical Chemistry, 84(14), 6054-6060. https://doi.org/ 10.1021/ac3008994.

127. Samouilov, A., Efimova, O. V., Bobko, A. A., Sun, Z., Petryakov, S., Eubank, T. D., Trofimov, D. G., Kirilyuk, I. A., Grigor'ev, I. A., Takahashi, W., Zweier, J. L., \& Khramtsov, V. V. (2014). In vivo proton-electron double-resonance imaging of extracellular tumor $\mathrm{pH}$ using an advanced nitroxide probe. Analytical Chemistry, 86(2), 1045-1052. https://doi.org/10.1021/ac402230h.

128. Komarov, D. A., Ichikawa, Y., Yamamoto, K., Stewart, N. J., Matsumoto, S., Yasui, H., Kirilyuk, I. A., Khramtsov, V. V., Inanami, O., \& Hirata, H. (2018). In vivo extracellular pH mapping of tumors using electron paramagnetic resonance. Analytical Chemistry, 90(23), 13938-13945. https://doi.org/10.1021/acs. analchem.8b03328.

129. Saha, G. B. (2010). Basics of PET imaging: physics, chemistry, and regulations (2nd ed.). New York: Springer.

130. Wyatt, L. C., Lewis, J. S., Andreev, O. A., Reshetnyak, Y. K., \& Engelman, D. M. (2017). Applications of pHLIP technology for cancer imaging and therapy. Trends in Biotechnology, 35(7), 653664. https://doi.org/10.1016/j.tibtech.2017.03.014.

131. Vāvere, A. L., Biddlecombe, G. B., Spees, W. M., Garbow, J. R., Wijesinghe, D., Andreev, O. A., et al. (2009). A novel technology for the imaging of acidic prostate tumors by positron emission tomography. Cancer Research, 69(10), 4510-4516. https://doi. org/10.1158/0008-5472.CAN-08-3781.

132. Demoin, D. W., Wyatt, L. C., Edwards, K. J., Abdel-Atti, D., Sarparanta, M., Pourat, J., Longo, V. A., Carlin, S. D., Engelman, D. M., Andreev, O. A., Reshetnyak, Y. K., Viola-Villegas, N., \& Lewis, J. S. (2016). PET imaging of extracellular $\mathrm{pH}$ in tumors with (64)Cu- and (18)F-labeled pHLIP peptides: a structure-activity optimization study. Bioconjugate Chemistry, 27(9), 2014-2023. https:// doi.org/10.1021/acs.bioconjchem.6b00306.

133. Flavell, R. R., Truillet, C., Regan, M. K., Ganguly, T., Blecha, J. E., Kurhanewicz, J., VanBrocklin, H. F., Keshari, K. R., Chang, C. J., Evans, M. J., \& Wilson, D. M. (2016). Caged [(18)F]FDG glycosylamines for imaging acidic tumor microenvironments using positron emission tomography. Bioconjugate Chemistry, 27(1), 170178. https://doi.org/10.1021/acs.bioconjchem.5b00584.

134. Keereweer, S., Van Driel, P. B., Snoeks, T. J., Kerrebijn, J. D., Baatenburg de Jong, R. J., Vahrmeijer, A. L., et al. (2013). Optical image-guided cancer surgery: challenges and limitations. Clinical Cancer Research, 19(14), 3745-3754. https://doi.org/10. 1158/1078-0432.CCR-12-3598.

135. Chen, L. Q., \& Pagel, M. D. (2015). Evaluating pH in the extracellular tumor microenvironment using CEST MRI and other imaging methods. Adv Radiol, 2015, 1-25. https://doi.org/10.1155/ 2015/206405

136. Martin, G. R., \& Jain, R. K. (1994). Noninvasive measurement of interstitial $\mathrm{pH}$ profiles in normal and neoplastic tissue using fluorescence ratio imaging microscopy. Cancer Research, 54(21), 5670-5674.

137. Robey, I. F., Baggett, B. K., Kirkpatrick, N. D., Roe, D. J., Dosescu, J., Sloane, B. F., Hashim, A. I., Morse, D. L., Raghunand, N., Gatenby, R. A., \& Gillies, R. J. (2009). Bicarbonate increases tumor $\mathrm{pH}$ and inhibits spontaneous metastases. Cancer Research, 69(6), 2260-2268. https://doi.org/10.1158/0008-5472.CAN-07-5575.

138. Lin, Y., Wu, T. Y., \& Gmitro, A. F. (2012). Error analysis of ratiometric imaging of extracellular $\mathrm{pH}$ in a window chamber model. Journal of Biomedical Optics, 17(4), 046004. https://doi. org/10.1117/1.JBO.17.4.046004.

139. Tsai, Y. T., Zhou, J., Weng, H., Shen, J., Tang, L., \& Hu, W. J. (2014). Real-time noninvasive monitoring of in vivo inflammatory responses using a $\mathrm{pH}$ ratiometric fluorescence imaging probe. Advanced Healtcare Materials, 3(2), 221-229. https://doi.org/10. 1002/adhm.201200365.
140. Takahashi, S., Kagami, Y., Hanaoka, K., Terai, T., Komatsu, T., Ueno, T., Uchiyama, M., Koyama-Honda, I., Mizushima, N., Taguchi, T., Arai, H., Nagano, T., \& Urano, Y. (2018). Development of a series of practical fluorescent chemical tools to measure $\mathrm{pH}$ values in living samples. Journal of the American Chemical Society, 140(18), 5925-5933. https://doi.org/10.1021/ jacs.8b00277.

141. Wang, Y., Zhou, K., Huang, G., Hensley, C., Huang, X., Ma, X., Zhao, T., Sumer, B. D., DeBerardinis, R. J., \& Gao, J. (2014). A nanoparticle-based strategy for the imaging of a broad range of tumours by nonlinear amplification of microenvironment signals. Nature Materials, 13(2), 204-212. https://doi.org/10.1038/ nmat3819.

142. Wang, L., Fan, Z., Zhang, J., Changyi, Y., Huang, C., Gu, Y., Xu, Z., Tang, Z., Lu, W., Wei, X., \& Li, C. (2015). Evaluating tumor metastatic potential by imaging intratumoral acidosis via $\mathrm{pH}$ activatable near-infrared fluorescent probe. International Journal of Cancer, 136(4), E107-E116. https://doi.org/10.1002/ijc.29153.

143. Ntziachristos, V., \& Razansky, D. (2010). Molecular imaging by means of multispectral optoacoustic tomography (MSOT). Chemical Reviews, 110(5), 2783-2794. https://doi.org/10.1021/ cr9002566.

144. Su, R., Ermilov, S., Liopo, A., \& Oraevsky, A. (2013). Laser OptoAcoustic tomography: towards new technology for biomedical diagnostics. Nuclear Instruments and Methods in Physics Research A, 720, 58-61. https://doi.org/10.1016/j.nima.2012.12.035.

145. Zackrisson, S., van de Ven, S. M., \& Gambhir, S. S. (2014). Light in and sound out: emerging translational strategies for photoacoustic imaging. Cancer Research, 74(4), 979-1004. https://doi.org/ 10.1158/0008-5472.CAN-13-2387.

146. Wang, L. V., \& Hu, S. (2012). Photoacoustic tomography: in vivo imaging from organelles to organs. Science, 335(6075), 1458 1462. https://doi.org/10.1126/science.1216210.

147. Oraevsky, A. A. (2015). Contrast agents for optoacoustic imaging: design and biomedical applications. Photoacoustics, 3(1), 1-2. https://doi.org/10.1016/j.pacs.2015.01.002.

148. Longo, D. L., Stefania, R., Callari, C., De Rose, F., Rolle, R., Conti, L., et al. (2017). Water soluble melanin derivatives for dynamic contrast enhanced photoacoustic imaging of tumor vasculature and response to antiangiogenic therapy. Advanced Healtcare Materials, 6(1). https://doi.org/10.1002/adhm.201600550.

149. Longo, D. L., Stefania, R., Aime, S., \& Oraevsky, A. (2017). Melanin-based contrast agents for biomedical optoacoustic imaging and theranostic applications. International Journal of Molecular Sciences, 18(8). https://doi.org/10.3390/ijms18081719.

150. Weber, J., Beard, P. C., \& Bohndiek, S. E. (2016). Contrast agents for molecular photoacoustic imaging. Nature Methods, 13(8), 639-650. https://doi.org/10.1038/nmeth.3929.

151. Chen, Q., Liu, X., Chen, J., Zeng, J., Cheng, Z., \& Liu, Z. (2015). A self-assembled albumin-based nanoprobe for in vivo ratiometric photoacoustic $\mathrm{pH}$ imaging. Advanced Materials, 27(43), 6820 6827. https://doi.org/10.1002/adma.201503194.

152. Chen, Q., Liu, X., Zeng, J., Cheng, Z., \& Liu, Z. (2016). AlbuminNIR dye self-assembled nanoparticles for photoacoustic $\mathrm{pH}$ imaging and $\mathrm{pH}$-responsive photothermal therapy effective for large tumors. Biomaterials, 98, 23-30. https://doi.org/10.1016/j. biomaterials.2016.04.041.

153. Miao, Q., Lyu, Y., Ding, D., \& Pu, K. (2016). Semiconducting oligomer nanoparticles as an activatable photoacoustic probe with amplified brightness for in vivo imaging of $\mathrm{pH}$. Advanced Materials, 28(19), 3662-3668. https://doi.org/10.1002/adma.201505681.

154. Jo, J., Lee, C. H., Kopelman, R., \& Wang, X. (2017). In vivo quantitative imaging of tumor $\mathrm{pH}$ by nanosonophore assisted multispectral photoacoustic imaging. Nature Communications, 8(1), 471. https://doi.org/10.1038/s41467-017-00598-1. 